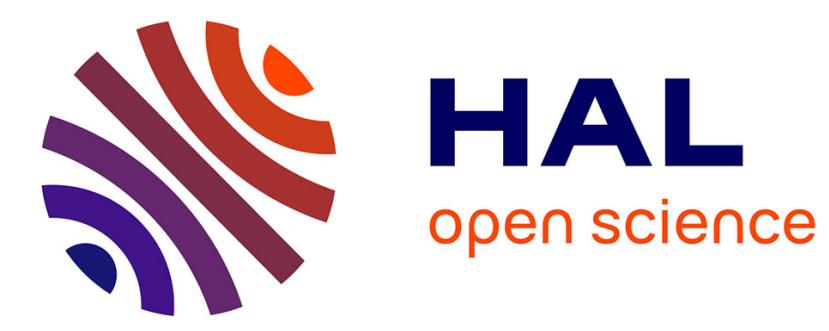

\title{
Secondary instability of the stably stratified Ekman layer
}

Nadia Mkhinini, Thomas Dubos, Philippe Drobinski

\section{To cite this version:}

Nadia Mkhinini, Thomas Dubos, Philippe Drobinski. Secondary instability of the stably stratified Ekman layer. Journal of Fluid Mechanics, 2013, 728 (August), pp.29-57. 10.1017/jfm.2013.250 . hal-01099546

\section{HAL Id: hal-01099546 \\ https://hal.science/hal-01099546}

Submitted on 4 Jan 2015

HAL is a multi-disciplinary open access archive for the deposit and dissemination of scientific research documents, whether they are published or not. The documents may come from teaching and research institutions in France or abroad, or from public or private research centers.
L'archive ouverte pluridisciplinaire HAL, est destinée au dépôt et à la diffusion de documents scientifiques de niveau recherche, publiés ou non, émanant des établissements d'enseignement et de recherche français ou étrangers, des laboratoires publics ou privés. 


\title{
Secondary instability of the stably stratified Ekman layer
}

\author{
Nadia Mkhinini $\dagger$, Thomas Dubos and Philippe Drobinski \\ Laboratoire de Météorologie Dynamique, École Polytechnique, 91120 Palaiseau, France \\ (Received 12 June 2012; revised 3 May 2013; accepted 13 May 2013; \\ first published online 1 July 2013)
}

The Ekman flow, an exact solution of the Boussinesq equations with rotation, is a prototype flow for both atmospheric and oceanic boundary layers. The effect of stratification on the finite-amplitude longitudinal rolls developing in the Ekman flow and their three-dimensional stability is studied by means of linearized and nonlinear numerical simulations. Similarities and differences with respect to billows developing in the Kelvin-Helmholtz $(\mathrm{KH})$ unidirectional stratified shear flow are discussed. Prandtl number effects are investigated as well as the role played by the buoyant-convective instability. For low Prandtl number, the amplitude of the saturated rolls vanishes at the critical bulk Richardson number, while at high Prandtl number, finite-amplitude rolls are found. The Prandtl number also affects how the growth rate of the secondary instability evolves as the Richardson number is increased. For low Prandtl number, the growth rate decreases as the Richardson number increases while it remains significant for large Prandtl number over the range of stratification studied. This behaviour is likely a result of the differing amplitudes of the roll vortices. Furthermore, the most unstable wave vector is much lower than for the secondary instability of KH billows. Examination of the energetics of the secondary instability shows that buoyant-convective instability is present locally at high Reynolds and Prandtl numbers but plays an overall minor role despite the presence in the base flow of statically unstable regions characterized by a high Richardson number.

Key words: boundary layer, instability, stratified flows

\section{Introduction}

The Ekman boundary layer was initially discovered as an explanation for the vertical variation of the ocean current near the sea surface (Ekman 1905). The classic Ekman profile is an exact solution to the Boussinesq equations with friction and Coriolis force assuming a constant kinematic viscosity. Its study has provided dynamical explanations of particular features of the atmospheric boundary layer, especially longitudinal roll vortices leading to the formation of cloud streets (Etling \& Brown 1993; Young et al. 2002). Like the atmospheric or oceanic boundary layers, which can experience near-neutral, convective or stably stratified conditions, the Ekman layer exists in nonstratified, unstably stratified or stably stratified variants. The dynamics of the Ekman layer are worth studying both as a generic flow forming near boundaries in rotating

$†$ Email address for correspondence: nadia.mkhinini@doc.polytechnique.org 
fluids, and as a minimal model allowing to study the structure of geophysical boundary layers resulting from purely dynamical effects, while ignoring other important driving factors such as, say, radiation.

The linear stability of the laminar, non-stratified Ekman flow over a smooth surface has been studied for quite some time, theoretically (Faller \& Kaylor 1966; Lilly 1966; Leibovich \& Lele 1985) and experimentally (Caldwell \& Van Atta 1970). Two different types of instability are present: the so-called 'parallel instability' (also called type II), which is the first to develop at Reynolds number $R e \simeq 54$, and an inflectionpoint instability (type I), which appears at $R e \simeq 113$ and becomes dominant at higher $R e$. Near the critical Reynolds number two-dimensional (2D) direct numerical simulations (DNS) and weakly nonlinear amplitude expansions have been used to predict the saturation of the instability into a finite amplitude roll travelling at a constant speed in a modified mean flow (Faller \& Kaylor 1966; Brown 1970; Chlond 1987; Haeusser \& Leibovich 2003). Dubos, Barthlott \& Drobinski (2008) study the secondary instability of these rolls. The turbulent regime has been studied mostly numerically (Coleman, Ferziger \& Spalart 1992; Coleman 1999; Spalart, Coleman \& Johnstone 2008, 2009).

Work on the effects of stratification is less complete. The effect on the linear instability is, as expected, that sufficiently strong stratification stabilizes the flow, resulting in a critical bulk Richardson number $R i$ above which the flow is linearly stable (Brown 1972; Kaylor \& Faller 1972). Coleman et al. (1992) explore the turbulent regime of the stratified Ekman layer. However the transition from the laminar flow to fully developed turbulence has not been studied in detail. In particular, the nonlinear saturation of the linear instability, and the instability of the resulting secondary flow, remain to be examined. In this work we study the secondary instability of the travelling rolls that develop as the primary instability saturates, and compare it with the secondary instability of Kelvin-Helmholtz (KH) billows, which has been studied in great detail (Peltier \& Caulfield 2003).

The case of KH billows in an unbounded stratified fluid suggests the occurrence of a violent secondary instability dominated by local buoyant-convective instability caused by overturning in the roll cores (Peltier \& Caulfield 2003). Such an overturning is also present in the rolls forming in the stratified Ekman layer (Dubos et al. 2008). In the Ekman layer, important additional dynamical ingredients are the veering of the mean wind profile, the Coriolis force and the bottom boundary, making the roll vortex flow more complex with axial velocity added to the cross-roll velocity, and hence three-dimensional (3D) shear. Therefore, our investigation here is focused in particular on how similar the secondary instabilities of KH billows and Ekman layer rolls are, and how important the role played by the convective instability is in the Ekman case. We furthermore explore Prandtl number effects, since varying the Prandtl number at fixed Reynolds number allows us to enhance the relative strength of buoyancy gradients compared with velocity gradients. One expects enhanced buoyancy gradients to amplify the potential role of the convective instability, as expressed for instance by the expression for the bulk Rayleigh number $R a_{b}=R e^{2} R i P r$.

A geophysical motivation for the study of the secondary instability of the stratified Ekman flow is the peculiar character of stably stratified boundary-layer turbulence as observed in the atmosphere. Indeed sporadic and non-stationary turbulence is observed in the regime classified as strongly stable based on the Monin-Obukhov similarity theory, while continuous, statistically stationary turbulence takes place at weaker stability (Mahrt 1999). According to the mechanisms involved in the generation of turbulence in a $\mathrm{KH}$ flow, the time scale of secondary instability should be dictated 
by local overturning while the emergence of a roll vortex generating this overturning should take place within an advective time scale. One may therefore speculate that the emergence (quiescent)/breakdown (turbulent) phases of the eddy life cycle should become increasingly asymmetric as stratification increases, with longer quiescent phases and shorter turbulent phases. The secondary instability of stratified Ekman layer roll vortices may therefore shed light on dynamical sources of turbulence intermittency, without invoking other causes that may be relevant depending on specific atmospheric and topographic conditions (e.g. gravity waves, drainage flows, meandering, interaction with the soil).

Despite the potential relevance for geophysical boundary layers, we do not attempt here to reproduce non-dimensional parameters found in the atmosphere or ocean. Indeed atmospheric Reynolds numbers $R e \backsim 10^{6}$ for oceanic Schmidt numbers of several hundreds are unreachable with our numerical tools. Instead we place ourselves in a range of Reynolds numbers where the inflexion-point instability of the Ekman layer dominates the primary instability, with a moderate Reynolds-number dependence. Our choice of control parameters is further guided by the desire to evaluate the potential role of convective instability in the secondary instability of stratified Ekman layer rolls. Varying the Prandtl number is therefore here a means to vary the respective strengths of velocity gradients and buoyancy gradients produced in the saturated rolls, and their dynamical effect: increasing $P r$ at constant $R e$ enhances buoyancy gradients while leaving velocity gradients roughly unchanged, increasing the likeliness of a convective secondary instability as indicated by a local Rayleigh number. Strong effects are already observed when varying $\operatorname{Pr}$ from 1 to 4 , and the present study is limited to these values.

In $\S 2$ the relevant equations and bulk parameters are defined, and the modification of the saturated Ekman rolls by a linear stratification is studied. Strong Prandtl number effects are observed. Notably the bifurcation at the critical Richardson number changes from a supercritical bifurcation at $\operatorname{Pr}=1$ to a subcritical bifurcation at $\operatorname{Pr}=4$. It is also found that the maximum local Rayleigh number is much higher at $\operatorname{Pr}=4$ than $\operatorname{Pr}=1$. The secondary instability developing over this new base flow is studied in $\S 3$. Stratification profoundly affects the characteristics of the secondary instability, and substantial differences with respect to the $\mathrm{KH}$ case are found with respect to the selected horizontal wavenumber and the energetics of the instability. In $\S 4$ results are summarized and discussed.

\section{Ekman rolls in a stratified environment}

In this section the emergence of longitudinal rolls in a stratified environment, following the primary instability studied by Lilly (1966) and Brown (1972), is described. We focus especially on the influence of the ambient stratification and of the Prandtl number of the fluid on the energetics of these KH-like vortices, the bifurcation near the critical bulk Richardson number and the appearance of unstably stratified regions within the vortex core.

\subsection{Equations and parameters}

We assume that the fluid motion is well described by the Boussinesq equations with kinematic viscosity $v$ and diffusivity $\kappa$. Depending on the fluid and flow of interest, $\kappa$ will be the kinematic diffusion coefficient for either heat or another quantity contributing to buoyancy such as salinity. The equations are written in dimensional form in a rotating reference frame with Coriolis parameter $f$, then made dimensionless 
in the same way as Brown (1972). Time, space, velocity, vorticity, buoyancy and pressure appear with a hat in the dimensional formulation and lose their hat in the non-dimensionalized form:

$$
\begin{gathered}
\frac{\partial \hat{\boldsymbol{U}}}{\partial \hat{t}}+\left(\hat{\omega}+f \boldsymbol{e}_{z}\right) \times \hat{\boldsymbol{U}}+\nabla \hat{H}-\widehat{b} \cdot \boldsymbol{e}_{z}=v \Delta \hat{\boldsymbol{U}}, \\
\frac{\partial \hat{b}}{\partial \hat{t}}+\hat{u} \frac{\partial \hat{b}}{\partial \hat{x}}+\hat{v} \frac{\partial \hat{b}}{\partial \hat{y}}+\hat{w} \frac{\partial \hat{b}}{\partial \hat{z}}+N^{2} \hat{w}=\kappa \Delta \hat{b} .
\end{gathered}
$$

In (2.1)-(2.2), $\hat{t}$ is the time, the gradient is relative to the position $\hat{\boldsymbol{x}}$ in 3D space, $\hat{H}$ is the Bernoulli function, $\hat{\boldsymbol{U}}=(\hat{u}, \hat{v}, \hat{w})$ is the flow velocity decomposed into horizontal components $\hat{u}$ and $\hat{v}$ and the vertical component $\hat{w}, \hat{\boldsymbol{\omega}}=\operatorname{curl} \hat{\boldsymbol{U}}$ is its vorticity. For buoyancy, the boundary condition at $z=0$ is that $\hat{B}$, hence $\hat{b}$, vanishes. The total buoyancy is

$$
\hat{B}=N^{2} z+\hat{b}(x, y, z, t)
$$

where $N$ is the Brunt-Väisälä frequency of the ambient linear stratification. The vertical direction is along $\boldsymbol{e}_{z}$. The boundary conditions are $\hat{\boldsymbol{U}}(\hat{z}=0)=0$ and $\hat{\boldsymbol{U}}(\hat{z}=\infty)=\boldsymbol{U}_{g}$ where $\boldsymbol{U}_{g}$ is the fluid velocity away from the lower boundary.

We scale velocities by $G=\left\|\hat{\boldsymbol{U}}_{g}\right\|$ and lengths by the laminar boundary-layer depth $\delta_{E}=\sqrt{2 \nu / f}$, hence we scale time by the advective time scale $\tau_{a d}=\delta_{E} / G$ :

$$
\begin{array}{rlrl}
\boldsymbol{U} & =\hat{\boldsymbol{U}} / G, & H & =\hat{H} / \rho G^{2}, \\
\boldsymbol{x} & =\hat{\boldsymbol{x}} / \delta_{E} & z & =\hat{z} / \delta_{E}, \\
t & =\hat{t} / \tau_{a d} & \boldsymbol{\omega} & =\tau_{a d} \hat{\boldsymbol{\omega}}, \\
& b & =\hat{b} / G N .
\end{array}
$$

The resulting non-dimensional equations are

$$
\begin{aligned}
& \operatorname{div} \boldsymbol{U}=0 \text {, } \\
& \frac{\partial \boldsymbol{U}}{\partial t}+\left(\omega+\frac{2}{\operatorname{Re}} \boldsymbol{e}_{z}\right) \times \boldsymbol{U}+\nabla H=\frac{1}{\operatorname{Re}} \Delta \boldsymbol{U}+\sqrt{\operatorname{Ri} b \boldsymbol{e}_{z}}, \\
& \frac{\partial b}{\partial t}+\boldsymbol{U} \cdot \nabla b=\frac{1}{\operatorname{RePr}} \Delta b-\sqrt{\operatorname{Ri} w},
\end{aligned}
$$

where

$$
R e=\frac{G \delta_{E}}{\nu}, \quad \operatorname{Pr}=\frac{v}{\kappa}, \quad R i=\frac{N^{2}}{\left(G / \delta_{E}\right)^{2}}
$$

are the Reynolds number, the Prandtl number and bulk Richardson number, respectively. The scaling of buoyancy is chosen in such a way that the nondimensional kinetic and potential energy can be expressed as

$$
K E=\frac{1}{2} \int_{0}^{\infty}\left\langle u^{2}+v^{2}+w^{2}\right\rangle_{x y} \mathrm{~d} z, \quad P E=\frac{1}{2} \int_{0}^{\infty}\left\langle b^{2}\right\rangle_{x y} \mathrm{~d} z
$$

where $\langle.\rangle_{x y}$ is a horizontal average. 

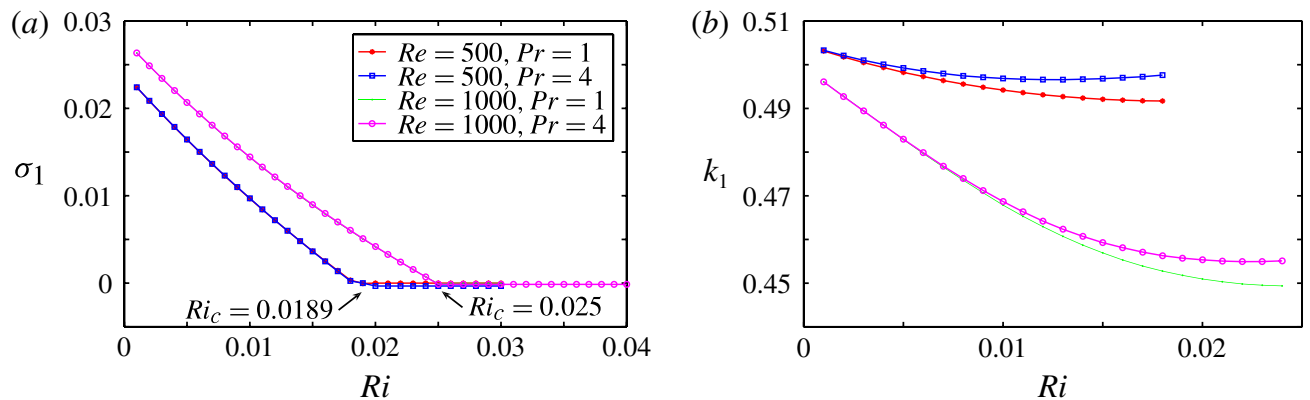

FIGURE 1. (Colour online) Growth rate $\sigma_{1}$ of the primary instability $(a)$ and its corresponding wave vector $k_{1}(b)$ as a function of the Richardson number $R i$ number for four pairs $(\operatorname{Re}, P r)$.

\subsection{Linear primary instability}

The Ekman flow is an exact steady-state and horizontally homogeneous solution of (2.1)-(2.2):

$$
\begin{aligned}
U_{0} & =1-\mathrm{e}^{-z} \cos z, \\
V_{0} & =\mathrm{e}^{-z} \sin z \\
W_{0}, B_{0} & =0
\end{aligned}
$$

Note that, due to our choice of a linear profile $N^{2} z$ for the background buoyancy in (2.3), equation (2.2) is exactly satisfied with $\kappa \neq 0$.

As studied by Lilly (1966) in the non-stratified case and by Brown (1972) in the stratified case, this flow is subject to a linear instability whose characteristics we recall now. Infinitesimal 2D perturbations added to the Ekman flow

$$
\begin{gathered}
\boldsymbol{U}(x, y, z, t)=\boldsymbol{U}_{0}(z)+\boldsymbol{U}_{2 D}(x, y, z, t) \\
b(x, y, z, t)=b_{0}(z)+b_{2 D}(x, y, z, t)
\end{gathered}
$$

evolve according to linearized equations whose solution can be sought as a superposition of exponentially growing eigenmodes:

$$
\boldsymbol{U}_{2 D}(x, y, z, t)=\mathrm{e}^{\sigma_{1} t} \cdot \mathrm{e}^{\mathrm{i} k_{1} \cdot x} \cdot \boldsymbol{U}_{2 \text { Deigen }}(z)+\text { c.c. }
$$

Given the global control parameters $\operatorname{Re}, \operatorname{Ri}, \operatorname{Pr}, \sigma_{1}\left(\boldsymbol{k}_{1}\right)$ is obtained for each horizontal wave vector $\boldsymbol{k}_{1}$ by solving numerically a linear eigenvalue problem (Dubos et al. 2008). We note $\boldsymbol{k}_{1}(R e, R i, P r)$ the most unstable wave vector.

In figure 1 we plot the growth rate $\sigma_{1}$ as a function of stratification for four pairs of $(R e, P r)$. This calculation starts at $R i=0$ where the most unstable mode is of so-called type II (inflection point instability; Lilly 1966). We then progressively increase the Richardson number while looking for a local maximum of the real part $\sigma_{1}$ in the vicinity of the previously found $\boldsymbol{k}_{1}(R e, R i, P r)$, effectively following the type II branch of instability. For fixed $(R e, P r)$ there is a critical value of $R i_{c}$ above which the linear instability is suppressed. This critical value depends on the Reynolds number but very little on the Prandtl number: for $R e=500, R i_{c} \simeq 0.0189$ and for $R e=1000$, $R i_{c} \simeq 0.025$. Those critical values are small compared with the Miles-Howard criterion $R i_{c}=1 / 4$ (Howard 1961; Miles 1961). As discussed by Brown (1972), the apparent discrepancy results from $R i$ being a bulk Richardson number, while the Miles criterion 
involves a local (gradient) Richardson number. In fact, the local Richardson number computed at the relevant inflection point is close to the theoretical upper value of $1 / 4$ when the bulk Richardson number reaches its critical value (Brown 1972).

After finding the most unstable mode, we redefine the axes by taking the $x$-axis parallel to $\boldsymbol{k}_{1}$. The Ekman stationary solution (2.10b) then becomes

$$
\begin{aligned}
& U_{E}(z)=\cos \varepsilon-\mathrm{e}^{-z} \cos (\varepsilon-z) \\
& V_{E}(z)=\sin \varepsilon-\mathrm{e}^{-z} \sin (\varepsilon-z)
\end{aligned}
$$

where $\varepsilon$ is the angle between the direction of the geostrophic flow and $\boldsymbol{k}_{1}$ (Leibovich $\&$ Lele 1985).

\subsection{Nonlinear saturation}

We now study the flow evolution after the perturbations have evolved for a long time. After initially small perturbations have grown exponentially for a sufficiently long time, they acquire a non-negligible amplitude and modify the flow, which takes a structure comparable with KH billows. Eventually the flow takes the form of equilibrated rolls moving with a constant phase speed $c$ without additional evolution. Figure 3 presents as an example the total buoyancy field of the roll obtained at $(\operatorname{Re}, \operatorname{Pr}, R i)=(500,4,0.015)$. The most important difference with respect to KH rolls is the existence of velocity along the roll axis. Another difference is that Ekman rolls are travelling states which are steady in a coordinate system moving with their phase velocity:

$$
\boldsymbol{U}(x, z, t)=\boldsymbol{U}_{E}(z)+\boldsymbol{U}_{2 D}(x-c t, z) .
$$

Therefore, their secondary stability can be studied in this moving reference frame. The KH billows, however, are constantly evolving. In order to circumvent the need to consider stability of a time-dependent flow, their secondary stability analysis is usually performed under the assumption of a frozen base flow, and the accuracy of this assumption needs to be verified a posteriori (Peltier \& Caulfield 2003).

In principle, equilibrated rolls can be obtained numerically by solving the timedependent equations (2.5)-(2.6) with a slightly perturbed initial condition. However, as discussed by Dubos et al. (2008) this is a long process and a more direct method, adopted here, is to solve for $\boldsymbol{U}(x, z)$ and $b(x, z)$ :

$$
\frac{\partial \boldsymbol{U}}{\partial t}+c \frac{\partial \boldsymbol{U}}{\partial x}=0, \quad \frac{\partial b}{\partial t}+c \frac{\partial b}{\partial x}=0 .
$$

In (2.15), $\partial \boldsymbol{U} / \partial t$ and $\partial b / \partial t$ are explicit functions of $\boldsymbol{U}(x, z), b(x, z)$ given by the Boussinesq equations (2.6)-(2.7). In order to eliminate the extra unknown $c$, we consider it an explicit function of $\boldsymbol{U}, b$ defined by

$$
c(\boldsymbol{U}, b)=-\frac{\int\left(\frac{\partial \boldsymbol{U}}{\partial x} \cdot \frac{\partial \boldsymbol{U}}{\partial t}+\frac{\partial b}{\partial x} \frac{\partial b}{\partial t}\right) \mathrm{d} x \mathrm{~d} y \mathrm{~d} z}{\int\left\|\frac{\partial \boldsymbol{U}}{\partial x}\right\|^{2}+\left(\frac{\partial b}{\partial x}\right)^{2} \mathrm{~d} x \mathrm{~d} y \mathrm{~d} z} .
$$

Indeed (2.15) implies that the desired $c$ satisfies (2.16). Equation (2.15) is then a nonlinear equation whose only unknowns are $\boldsymbol{U}, b$.

The spatial discretization is of the Galerkin type with $\boldsymbol{U}$ and $b$ belonging to suitable finite-dimensional functional spaces $\mathscr{U}$ and $\mathscr{B}$ (see Appendix). Equation (2.15) then becomes a system of nonlinear equations whose unknowns are the coefficients of 


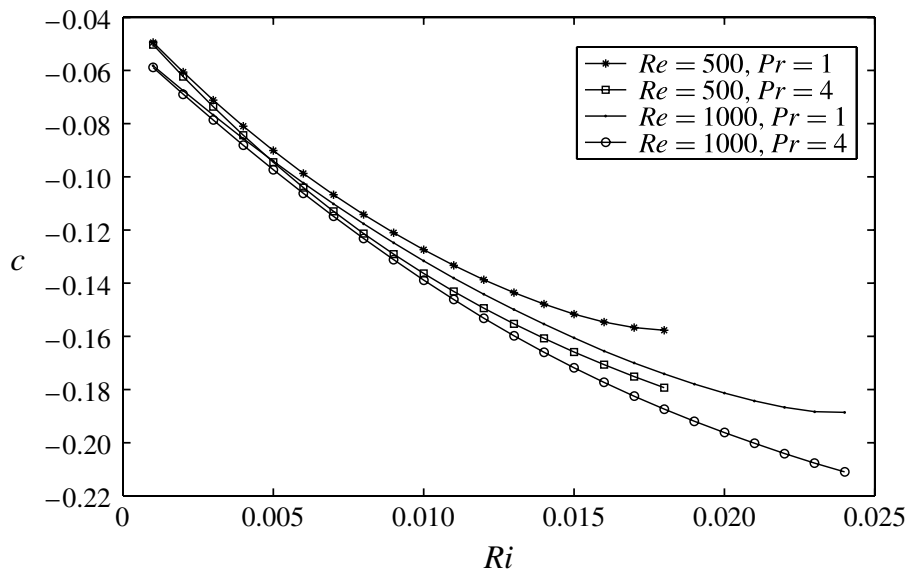

FIGURE 2. Phase velocity $c$ as a function of Richardson number Ri number for four pairs $(\operatorname{Re}, \operatorname{Pr})$.

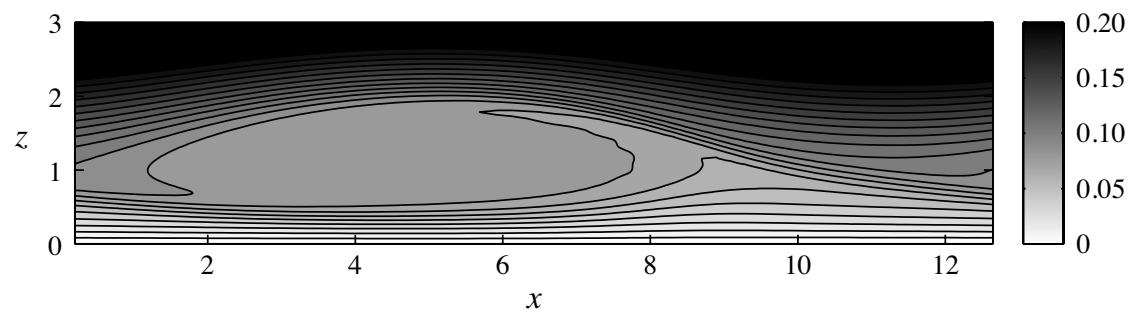

FIGURE 3. The $y$-averaged buoyancy in the form of saturated rolls moving with constant phase speed $c$ for parameters $(R e, P r, R i)=(1000,1,0.015)$ : buoyancy $R e=500, P r=$ $4, R i=0.015$.

the decomposition of $\boldsymbol{U}$ and $b$ on bases of $\mathscr{U}$ and $\mathscr{B}$. This system is solved by a Newton-Krylov iterative method (Dubos et al. 2008). Problem (2.15) is solved first at small Richardson number using a non-stratified solution as an initial guess of the iterative procedure. The Richardson number $R i$ is then gradually increased, each solution of iterations (2.15) being used as an initial guess to compute the travelling roll for the immediately larger $R i$. Figure 2 displays the evolution of the phase velocity as $R i$ is increased for $R e=500,1000$ and $\operatorname{Pr}=1,4$.

To analyse the effect of stratification on the equilibrated rolls, we plot in figure 4 their kinetic energy $K E$, potential energy $P E$ and total energy $T E=P E+K E$ as a function of Richardson number for four pairs of parameters $(R e, P r)$. For $\operatorname{Pr}=1$, the kinetic energy decreases continuously when the stratification increases. This is consistent with the increasing inhibition of vertical motion by the ambient stratification, already manifest in the decrease of the growth rate $\sigma_{1}$ with increasing $R i$. On the other hand, the potential energy grows slightly for weak stratifications and reaches a maximum near $R i=0.0075$ for $R e=500$ and near $R i=0.0125$ for $R e=1000$. For higher $R i$ potential energy eventually decreases until it vanishes for numbers of the Richardson number close to the critical Richardson number. The initial growth of the potential energy for the low values of $R i$ can be understood by the 


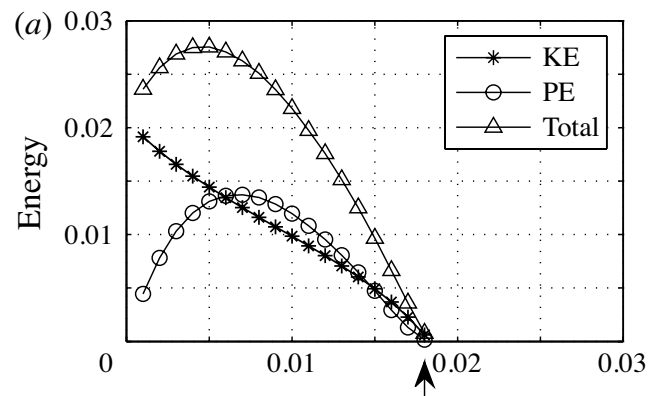

(b)
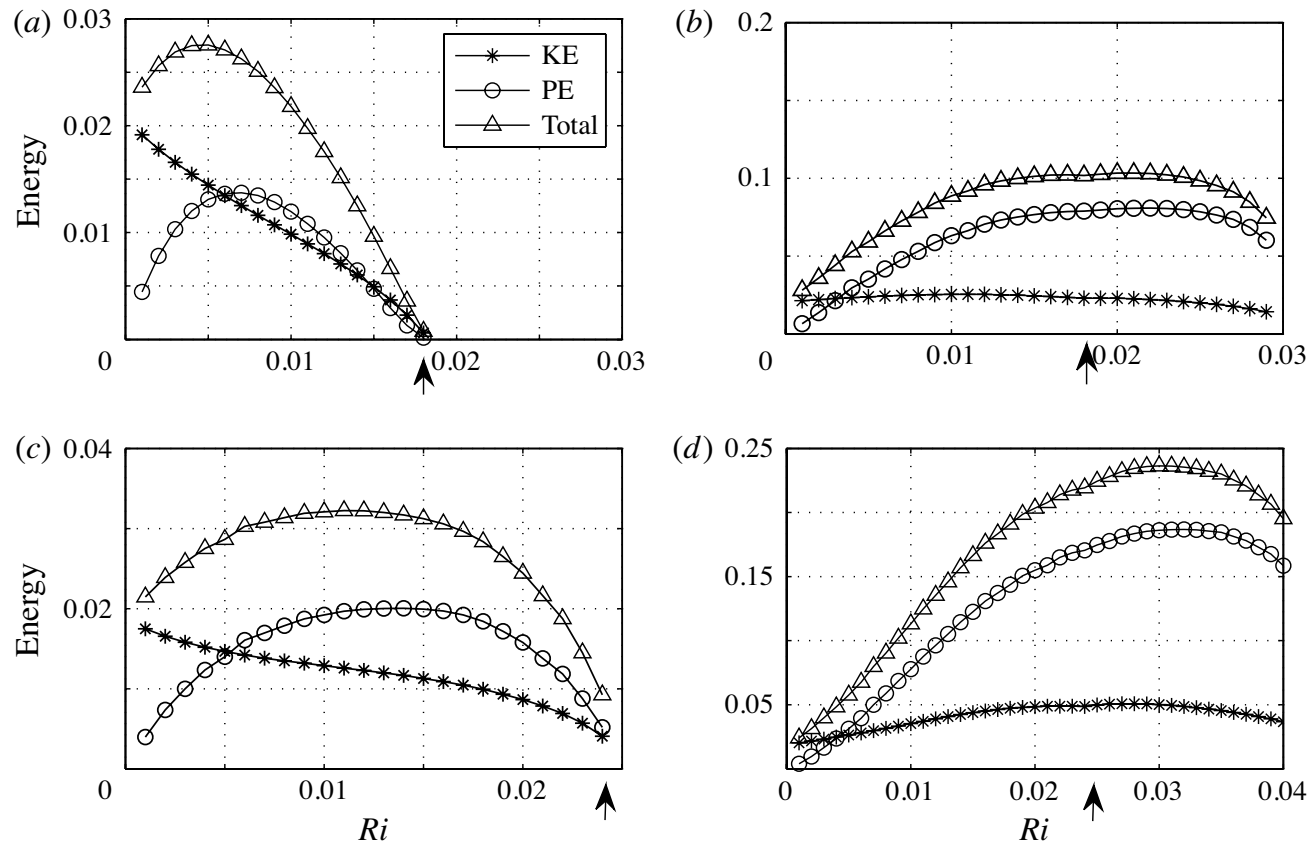

FIGURE 4. Kinetic, potential and total energy of equilibrated rolls as a function of Richardson number $R i$ for several $(R e, P r)$. Arrows point at the value of $R i_{c}$ : (a) $\operatorname{Re}=500$ and $\operatorname{Pr}=1$; (b) $\operatorname{Re}=500$ and $\operatorname{Pr}=4$; (c) $\operatorname{Re}=1000$ and $\operatorname{Pr}=1$; (d) $\operatorname{Re}=1000$ and $\operatorname{Pr}=4$.

fact that rolls remain rather strong and can extract potential energy from the stratified environment by lifting heavy fluid, as is apparent in figure 3 . When the stratification increases, rolls weaken substantially and become unable to lift heavy fluid, and their energy (kinetic and potential) eventually vanishes. The total energy TE follows the same behaviour of growth then decay with increasing Richardson number as the potential energy. The growth of $T E$ with increasing $R i$ for small $R i$ shows that the potential energy due to lifting of heavy fluid is, in this regime, larger than the deficit of kinetic energy due to a less strong vortex.

For $\operatorname{Pr}=4$, the kinetic energy does not decrease immediately as stratification is increased. Furthermore the kinetic energy remains finite when the Richardson number reaches $R i_{c}$. In terms of bifurcation theory of dynamical systems, this shows that the bifurcation at $R i=R i_{c}$ for $\operatorname{Pr}=4$ is of the subcritical type. Conversely the bifurcation is of supercritical type at $\operatorname{Pr}=1$. This implies that the nonlinear terms of (2.1)-(2.2) have a destabilizing effect at $P r=4$ which leads to a finite roll amplitude at $R i=R i_{c}$, while the nonlinear terms have a stabilizing effect for $\operatorname{Pr}=1$, leading to a vanishing roll amplitude at $R i=R i_{c}$. Previous work on the Ekman layer stability diagram has characterized the bifurcation in the non-stratified situation only. Haeusser \& Leibovich (2003) and Dubos et al. (2008) find a supercritical bifurcation when the Reynolds number reaches its critical value. The subcritical bifurcation observed at $\operatorname{Pr}=4$ allows us to compute equilibrated vortices at $R i>R i_{c}$. For this we progressively increase $R i$ and keep the wave vector constant at $\boldsymbol{k}_{1}=\boldsymbol{k}_{1}\left(R i=R i_{c}\right)$. We have been able to find solutions of (2.15) for $R i$ as large as almost twice $R i_{c}$. The maximum kinetic energy is 

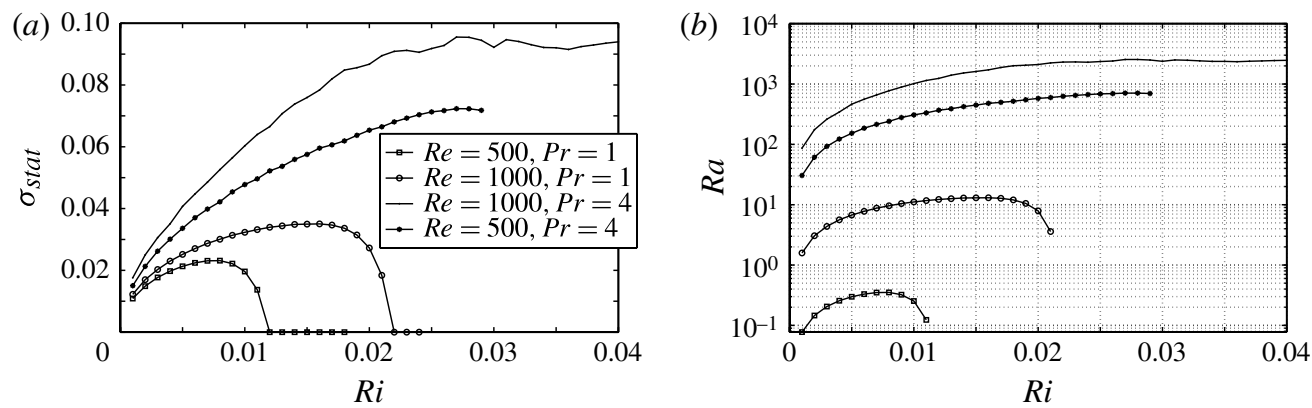

FIGURE 5. Maximum growth rate $\sigma_{\text {stat }}^{\max }$ of convective instability ( $a$ ) and Rayleigh number $R a$ (b) as a function of Richardson number $R i$ for the same values of $(R e, P r)$.

obtained for $R i>R i_{c}$. Over the whole range of $R i$ where rolls exist, we observe that their potential energy strongly dominates their kinetic energy.

Peltier \& Caulfield (2003) attributed the breakdown of KH vortices by secondary instability to buoyant-convective instability. To evaluate the potential for a buoyantconvective secondary instability we consider the total vertical buoyancy gradient.

$$
\hat{\sigma}_{\text {stat }}^{2}(\hat{x}, \hat{z} ; \operatorname{Re}, \operatorname{Pr}, R i)=-N^{2}-\frac{\partial}{\partial \hat{z}} \hat{b}(\hat{x}, \hat{z} ; \operatorname{Re}, \operatorname{Pr}, \operatorname{Ri}) .
$$

The flow is statically unstable where $\hat{\sigma}_{\text {stat }}^{2}>0$. In non-dimensional terms we have $\hat{\sigma}_{\text {stat }}=\sigma_{\text {stat }} G / \delta_{E}$ where

$$
\sigma_{s t a t}(x, z ; R e, R i, P r)=\sqrt{-\sqrt{R i} \frac{\partial b}{\partial z}-R i} .
$$

Figure 5(a) shows $\sigma_{\text {stat }}^{\max }(\operatorname{Re}, \operatorname{Pr}, R i)=\max _{x, z} \sigma_{\text {stat }}(x, z ; R e, P r, R i)$, the maximum value of $\sigma_{\text {stat }}$, as a function of $R i$, for four pairs of $(R e, P r)$. For $\operatorname{Pr}=1, \sigma_{\text {stat }}^{\max }(R i)$ evolves in a way similar to $P E(R i)$ in figure 4. Indeed, both potential energy and $\sigma_{\text {stat }}^{\max }$ reach their maximum value for a comparable value of $R i(R i=0.06$ at $R e=500$ and $R i=0.015$ at $R e=1000)$. For $P r=4$ the value of $\sigma_{\text {stat }}^{\max }$ is much bigger than its value for $\operatorname{Pr}=1$. Also, it does not reach a maximum over the range of Richardson numbers for which we were able to compute equilibrated rolls, at both $R e=500$ and $R e=1000$. Compared with the growth rate $\sigma_{1} \simeq 0.02$ of the primary instability, the value of $\sigma_{\text {stat }}^{\max }$ is larger than $\sigma_{1}$ for $\operatorname{Pr}=1$, and much larger than $\sigma_{1}$ for $\operatorname{Pr}=4$. This and the large value of the roll potential energy compared to its kinetic energy suggest that a fast convective secondary instability should be able to occur for $\operatorname{Pr}=4$, and maybe also for $\operatorname{Pr}=1$.

In order to assess the effect of viscosity and diffusion, which can prevent the development of a buoyant-convective instability even if $\sigma_{\text {stat }}^{2}>0$, we compute a Rayleigh number at each abscissa $x$ for each equilibrated roll as

$$
R a=\operatorname{Re}^{2} \cdot \operatorname{Pr} \cdot \sqrt{R i} \cdot \triangle B \cdot \delta_{\text {stat }}^{3}
$$

where $\delta_{\text {stat }}(x)=z_{\max }(x)-z_{\min }(x)$ is the thickness of the statically unstable region in which $\sigma_{\text {stat }}^{2}>0$ and $\Delta B=B\left(z_{\min }\right)-B\left(z_{\max }\right)$ is the difference in total buoyancy $B=z \sqrt{R i}+b$ between the top $z=z_{\max }$ and the bottom $z=z_{\min }$ of that statically unstable region. The maximum value of $R a$ is retained for each computed roll. Figure 5(b) displays the Rayleigh number computed for the same couples of $(\operatorname{Re}, \operatorname{Pr})$ 
studied before as a function of stratification. For $(\operatorname{Re}, \operatorname{Pr})=(1000,4)$, the values of $R a$ exceeds its critical value which is around $R a_{c} \sim 1000-2000$ depending on boundary conditions (Chandrasekhar 1961). According to this criterion, we expect a buoyantconvective secondary instability to develop for $(\operatorname{Re}, P r)=(1000,4)$. However, for the three other couples $(R e, P r)$, we expect the lower $R a$ to prevent the development of a buoyant-convective secondary instability.

\section{Secondary instability}

In $\S 2$ we have characterized the steady travelling rolls that emerge from the primary instability of the stratified Ekman flow. In this section we study their stability with respect to 3D perturbations, first in the linear approximation for infinitesimal perturbations and then in the nonlinear regime. For this we place ourselves in the frame moving with their phase speed $c$, in which rolls are exact steady-state solutions of the Boussinesq equations. We focus here on $R e=1000$, for two different Prandtl number $(P r=1$ and $P r=4)$. Unless otherwise mentioned, the results for $R e=500$ are close to those for $R e=1000$. We first perform a linear secondary stability analysis of the travelling rolls. We find that the unstable modes present some qualitative similarities with the $\mathrm{KH}$ case, but with important differences. In order to assess the importance of the convective instability, the energetics of the secondary instability are investigated. The nonlinear development of the secondary instability, the ensuing modification of the mean flow and the corresponding energetics are also investigated.

\subsection{Linear secondary instability}

We now consider the evolution of infinitesimal perturbations added to the rolls considered as a new basic flow:

$$
\begin{aligned}
\boldsymbol{U}(x, y, z, t) & =\boldsymbol{U}_{E}(z)+\boldsymbol{U}_{2 D}(x, z)+\boldsymbol{U}_{3 D}(x, y, z, t) \\
b(x, y, z, t) & =b_{E}(z)+b_{2 D}(x, z)+b_{3 D}(x, y, z, t)
\end{aligned}
$$

As for the primary instability, these $3 \mathrm{D}$ perturbations can be written as a superposition of eigenmodes

$$
\boldsymbol{U}_{3 D}(x, y, z, t)=\mathrm{e}^{\left(\sigma_{2}+\mathrm{i} \omega_{2}\right) t} \cdot \mathrm{e}^{\mathrm{i} k y \cdot y} \cdot \boldsymbol{U}_{3 D_{\text {eigen }}}(x, z)+\text { c.c. }
$$

where $\left(\sigma_{2}+\mathrm{i} \omega_{2}\right)$ is the complex growth rate of 3D perturbations, $k_{y}$ is the secondary wave vector along the $y$ direction and c.c. denotes complex conjugate. In this following we discuss only its real part $\sigma_{2}$. In full generality the secondary wave vector should also have a component along the $x$-axis. However, Dubos et al. (2008) have found in the non-stratified case that the secondary growth rate $\sigma_{2}$ depends very little on this component along $x$. We consider here as well only a secondary wave vector purely along the $y$-axis. We have not studied a possible dependence of $\sigma_{2}$ on an $x$-component of the secondary wave vector. However, we have checked in our subsequent nonlinear simulations that the growth rate and spatial structure of the emerging fastest-growing mode match those computed here. Therefore, the fastest-growing modes have indeed their secondary wave vector parallel to the $y$-axis. The same result was found for the secondary instability of stratified KH vortices (Klaassen \& Peltier 1985).

In order to obtain $\sigma_{2}$ numerically, the Boussinesq equations linearized around the basic flow $\boldsymbol{U}_{E}(z)+\boldsymbol{U}_{2 D}(x, z)$ are integrated forward in time for a time $T$ starting from an arbitrary initial condition. A post-processing Krylov method is then applied (Julien, Ortiz \& Chomaz 2004). The method is typically able to capture eigenmodes with growth rate of order $1 / T$ or larger (see Appendix). 

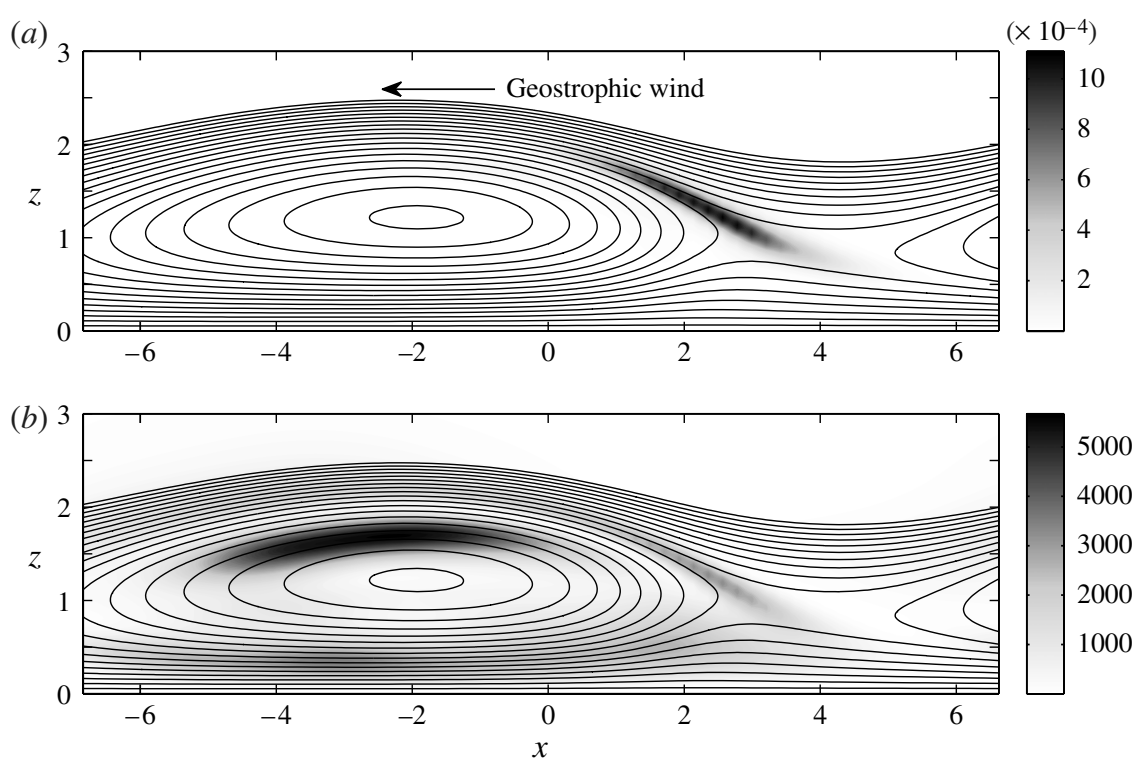

FIGURE 6. The $y$-averaged energy of two unstable eigenmodes. Black lines are the streamlines: (a) $R e=1000, R i=0.015, \operatorname{Pr}=4, k_{y}=2.08$; (b) $\operatorname{Re}=1000, R i=0.015$, $\operatorname{Pr}=4, k_{y}=0.36$.

Two typical unstable modes computed for two different wave vectors are displayed in figure 6 for $(R e, R i, P r)=(1000,0.015,4)$. The black lines are stream lines of the basic flow and the grey shade is the $y$-averaged total energy of normal modes in arbitrary units. Figure $6(a)$ corresponds to $k_{y}>1$. For this unstable mode the energy is concentrated near the hyperbolic stagnation point, as was found in the non-stratified case (Dubos et al. 2008). Non-stratified KH billows also develop a 3D instability concentrated near the hyperbolic stagnation point (Caulfield \& Peltier 2000). Figure $6(b)$ corresponds to $k_{y}<1$. Now the energy is concentrated along a closed streamline inside the vortex core. This is similar to the structure of the most unstable modes developing over stratified KH billows (Caulfield \& Peltier 2000).

Figure 7 presents the growth rate $\sigma_{2}$ of infinitesimal 3D perturbations as a function of $k_{y}$ for different strengths of stratification. The parameters that we present are $R e=1000$ for both $P r=1$ and $P r=4$. Several branches show up on the stability diagram. Broadly speaking, an isolated branch is found for $k_{y}>1$ and a number of other branches for $k_{y}<1$. Figure $7(c, d)$ is a zoom of figure $7(a, b)$ focusing on the range $0<k_{y}<0.5$. For $R i=0$, we recover at $k_{y}>1$ the unstable mode identified by Dubos et al. (2008). The dominant eigenmode is found in the branch $k_{y}>1$ for $\operatorname{Pr}=1, R i=(0,0.005)$ and for $\operatorname{Pr}=4, R i=(0,0.005,0.010)$. Furthermore, for $\operatorname{Pr}=1$, the modes for $k_{y}>1$ become less unstable as Richardson number increases and become stable for $R i \geqslant 0.010$ (figure $7 a, c$ ). For these larger values of $R i$ the dominant mode is found in the range $k_{y}<1$. This is at stark contrast with the secondary instability of $\mathrm{KH}$ billows, for which the fastest growing mode has a large wavenumber, which furthermore grows as $R i$ is increased (Caulfield \& Peltier 2000). For $\operatorname{Pr}=4$, the modes for $k_{y}>1$ do not have a monotonic behaviour as $R i$ increases. Nevertheless as the Richardson number becomes sufficiently high $(R i=0.015)$, the 



FIGURE 7. Secondary growth rate $\sigma_{2}$ as a function of secondary wavenumber $k_{y}$ for different levels of stratification, for $\operatorname{Pr}=1,4$ and $\operatorname{Re}=1000$ : $(a) \operatorname{Re}=1000, \operatorname{Pr}=1$; $(b)$ $\operatorname{Re}=1000, \operatorname{Pr}=4$; $(c) \operatorname{Re}=1000, \operatorname{Pr}=1 ;(d) \operatorname{Re}=1000, \operatorname{Pr}=4$. Panels $(c, d)$ are enlarged views of $(a, b)$, respectively, focusing on the range $0<k_{y}<0.5$.

most unstable mode is found in the range $k_{y}<1$ (figure $7 b, d$ ), as happens for $\operatorname{Pr}=1$ beyond $R i=0.010$.

Let us stress that the maximum secondary growth rate remains significant at $\sigma_{2} \simeq 0.025$ for $R i=0.015$ and $\operatorname{Pr}=4$ while for $\operatorname{Pr}=1$ and the same $R i, \sigma_{2}$ has declined to $\sigma_{2} \simeq 0.01$. This behaviour may be related to the fact that the roll vortices weaken as $R i$ approaches its critical value if $\operatorname{Pr}=1$, but not if $\operatorname{Pr}=4$.

\subsection{Energetics of the secondary instability}

To understand the sources of the energy of the 3D perturbations and how they modify the $y$-mean flow, we decompose the total flow into three parts, Ekman flow, 2D rolls and $3 \mathrm{D}$ perturbations,

$$
\begin{aligned}
& \boldsymbol{U}(x, y, z, t)=\boldsymbol{U}_{E}(z)+\boldsymbol{U}_{2 D}(x, z, t)+\boldsymbol{U}_{3 D}(x, y, z, t) \\
& \boldsymbol{b}(x, y, z, t)=\boldsymbol{b}_{E}(z)+\boldsymbol{b}_{2 D}(x, z, t)+\boldsymbol{b}_{3 D}(x, y, z, t)
\end{aligned}
$$

Note that in (3.1)-(3.2), $\boldsymbol{U}_{3 D}, b_{3 D}$ are infinitesimal, and $\boldsymbol{U}_{2 D}, b_{2 D}$ is a time-independent base flow while in (3.4) the $3 \mathrm{D}$ perturbations are finite and $\boldsymbol{U}_{2 D}, b_{2 D}$ is a timedependant $y$-averaged flow. When the 3D perturbations reach a finite amplitude, we define

$$
\begin{aligned}
\boldsymbol{U}_{E}(z)+\boldsymbol{U}_{2 D}(x, z, t) & =\langle\boldsymbol{U}(x, y, z, t)\rangle_{y}, \quad\left\langle\boldsymbol{U}_{3 D}(x, y, z, t)\right\rangle_{y}=0, \\
b_{E}(z)+b_{2 D}(x, z, t) & =\langle b(x, y, z, t)\rangle_{y}, \quad\left\langle b_{3 D}(x, y, z, t)\right\rangle_{y}=0 .
\end{aligned}
$$

We compute for each part the kinetic energy $K E$ and the potential energy $P E$ and so we form three reservoirs of energy. The fluxes appearing in each energy budget allow us to analyse the interactions between the three reservoirs. 
With decomposition (3.4) we can compute the kinetic energy $\left(K E_{3 D}\right)$ of $3 \mathrm{D}$ perturbations:

$$
\begin{aligned}
K E_{3 D} & =\left\langle\frac{u_{3 D}^{2}}{2}+\frac{v_{3 D}^{2}}{2}+\frac{w_{3 D}^{2}}{2}\right\rangle_{x, y, z} \\
\frac{\partial}{\partial t} K E_{3 D} & =S_{2 D-3 D}+S_{E-3 D}-X_{3 D}-\epsilon_{3 D} .
\end{aligned}
$$

In (3.7) $S_{2 D-3 D}$ is the production of 3D kinetic energy that results from the interaction between rolls and $3 \mathrm{D}$ perturbations, $S_{E-3 D}$ is the production that results from the interaction between the Ekman flow and 3D perturbations, $X_{3 D}$ is the exchange between kinetic and potential energy of 3D perturbations and $\epsilon_{3 D}$ is the dissipation of $K E_{3 D}$ :

$$
\begin{aligned}
S_{2 D-3 D} & =\left\langle\frac{\partial}{\partial x_{i}} u_{j, 2 D} u_{i, 3 D} u_{j, 3 D}\right\rangle_{x, y, z} \\
S_{E-3 D} & =\left\langle\frac{\partial}{\partial x_{i}} u_{j, E} u_{i, 3 D} u_{j, 3 D}\right\rangle_{x, y, z} \\
X_{3 D} & =-\sqrt{R i}\left\langle w_{3 D} b_{3 D}\right\rangle_{x, y, z} \\
\epsilon_{3 D} & =\frac{1}{R e}\left\langle\left\|\nabla u_{3 D}\right\|^{2}+\left\|\nabla v_{3 D}\right\|^{2}+\left\|\nabla w_{3 D}\right\|^{2}\right\rangle_{x, y, z} .
\end{aligned}
$$

In (3.8)-(3.9), a summation is implied on indices $i=1,2,3$ and $j=1,2,3$ following Einstein's notation and $\left(u_{E}, v_{E}, w_{E}\right)=\left(u_{1, E}, u_{2, E}, u_{3, E}\right)$ (and similar conventions apply to $u_{i, 2 D}$ and $u_{i, 3 D}$ ). The factor $\sqrt{R i}$ in (3.10) results from our convention for scaling the buoyancy. Using the energy budget (3.7) we can define a secondary growth rate $\sigma_{2}^{K E}$ and decompose it as a sum of contributions from the various interactions:

$$
\sigma_{2}^{K E}=\frac{1}{2} \frac{\frac{\partial}{\partial t} K E_{3 D}}{K E_{3 D}}=\sigma_{S_{3 D}}-\sigma_{X_{3 D}}-\sigma_{\epsilon_{3 D}}
$$

where $\sigma_{S_{3 D}}=\left(S_{2 D-3 D}+S_{E-3 D}\right) / K E_{3 D}, \sigma_{X_{3 D}}=X_{3 D} / K E_{3 D}$ and $\sigma_{\epsilon_{3 D}}=\epsilon_{3 D} / K E_{3 D}$. Here $\sigma_{2}^{K E}$ is defined in both nonlinear and linear regimes, and coincides with $\sigma_{2}$ in the linear regime.

Analogous budgets and decomposition can be written based on the potential energy $\left(P E_{3 D}\right)$ of 3D perturbations:

$$
\begin{aligned}
P E_{3 D} & =\left\langle\frac{b_{3 D}^{2}}{2}\right\rangle_{x, y, z} \\
\frac{\partial}{\partial t} P E_{3 D} & =\mathscr{H}_{2 D-3 D}+X_{3 D}-\mu_{3 D} .
\end{aligned}
$$

In (3.14) $\mathscr{H}_{2 D-3 D}$ is the production that results from the interaction between rolls and 3D perturbations, and $\mu_{3 D}$ is the dissipation of $P E_{3 D}$ due to diffusion:

$$
\begin{aligned}
\mathscr{H}_{2 D-3 D} & =-\left\langle u_{3 D} b_{3 D} \cdot \frac{\partial b_{2 D}}{\partial x}\right\rangle_{x, y, z}-\left\langle b_{3 D} w_{3 D} \cdot \frac{\partial b_{2 D}}{\partial z}\right\rangle \\
\mu_{3 D} & =\frac{1}{\operatorname{Re} \cdot \operatorname{Pr}}\left\langle\left\|\nabla b_{3 D}\right\|^{2}\right\rangle_{x, y, z} .
\end{aligned}
$$


By the same way the growth rate of $3 \mathrm{D}$ perturbations is decomposed as

$$
\sigma_{2}^{P E}=\frac{1}{2} \frac{\frac{\partial}{\partial t} P E_{3 D}}{P E_{3 D}}=\sigma_{\mathscr{H}_{2 D-3 D}}+\sigma_{X_{3 D}}-\sigma_{\mu_{3 D}},
$$

where $\sigma_{\mathscr{H}_{2 D-3 D}}=\mathscr{H}_{2 D-3 D} / P E_{3 D}, \sigma_{X_{3 D}}=X_{3 D} / P E_{3 D}$ and $\sigma_{\mu_{3 D}}=\mu_{3 D} / P E_{3 D}$.

The energetics of the $3 \mathrm{D}$ perturbations will be used to investigate the linear stage of the secondary instability. In the nonlinear stage the mean flow is modified. We analyse this modification through the evolution of the kinetic energy $\left(K E_{2 D}\right)$ and potential energy $\left(P E_{2 D}\right)$ of rolls:

$$
\begin{aligned}
K E_{2 D} & =\left\langle\frac{u_{2 D}^{2}}{2}+\frac{v_{2 D}^{2}}{2}+\frac{w_{2 D}^{2}}{2}\right\rangle_{x, y, z} \\
\frac{\partial}{\partial t} K E_{2 D} & =S_{2 D-3 D}+S_{E-2 D}-X_{2 D}-\epsilon_{2 D} .
\end{aligned}
$$

In (3.19) $S_{E-2 D}$ is the production that results from the interaction between the Ekman flow and $2 \mathrm{D}$ rolls, $X_{2 D}$ is the exchange between kinetic and potential energy for $2 \mathrm{D}$ rolls and $\epsilon_{2 D}$ is the dissipation of $K E_{2 D}$ :

$$
\begin{aligned}
S_{E-2 D}= & \left\langle 2 u_{2 D} u_{E} \frac{\partial u_{2 D}}{\partial x}+w_{2 D} u_{E} \cdot\left(\frac{\partial u_{2 D}}{\partial z}+\frac{\partial w_{2 D}}{\partial x}\right)\right\rangle_{x, y, z} \\
& +\left\langle\left(u_{2 D} v_{E}+u_{E} v_{2 D}\right) \cdot \frac{\partial v_{2 D}}{\partial x}+w_{2 D} v_{E} \frac{\partial v_{2 D}}{\partial z}\right\rangle_{x, y, z} \\
X_{2 D}= & -\sqrt{R i}\left\langle w_{2 D} b_{2 D}\right\rangle_{x, y, z} \\
\epsilon_{2 D}= & \frac{1}{R e}\left\langle\left\|\nabla u_{2 D}\right\|^{2}+\left\|\nabla v_{2 D}\right\|^{2}+\left\|\nabla w_{2 D}\right\|^{2}\right\rangle_{x, y, z} .
\end{aligned}
$$

Finally $P E_{2 D}$ can be decomposed as

$$
\begin{aligned}
P E_{2 D} & =\left\langle\frac{b_{2 D}^{2}}{2}\right\rangle_{x, y, z} \\
\frac{\partial}{\partial t} P E_{2 D} & =\mathscr{H}_{2 D-3 D}+X_{2 D}-\mu_{2 D} .
\end{aligned}
$$

In (3.24) $\mu_{2 D}$ is the dissipation of $P E_{2 D}$.

$$
\mu_{2 D}=\frac{1}{\operatorname{RePr}}\left\langle\left\|\nabla b_{2 D}\right\|^{2}\right\rangle_{x, y, z} .
$$

\subsection{Energetics in the linear stage of secondary instability}

The results we present now are based on fully nonlinear time integrations starting with the most unstable 3D mode previously identified added to the $2 \mathrm{D}$ rolls studied in $\S 2$. The 3D perturbations are given an initially small amplitude so that their dynamics are approximately linear at the beginning of the simulations. We show in figure 8 the budgets of 3D kinetic and potential energy for $R e=1000, R i=0.015$ and $\operatorname{Pr}=1,4$. Below we mention the times at which various events occur at $\operatorname{Pr}=1$ and $\operatorname{Pr}=4$ in order to discuss the sequence of these events and their energetics, but do not attach a particular importance to the timings themselves. In particular, there is no reason for the 

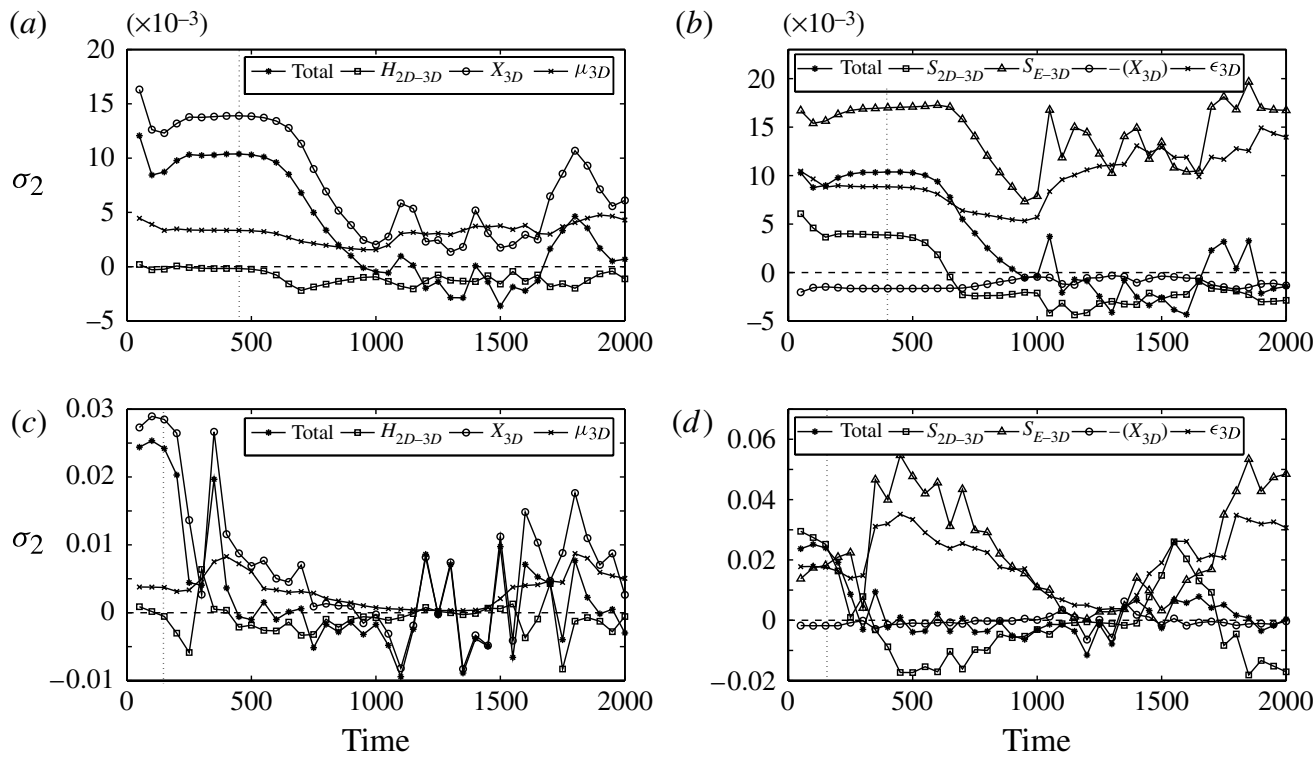

FIGURE 8. Energy budget of 3D perturbations for $R e=1000, R i=0.015$ and $\operatorname{Pr}=1,4$ : $(a, c)$ potential energy budget; $(b, d)$ kinetic energy budget; $(a, b) \operatorname{Pr}=1 ;(c, d) \operatorname{Pr}=4$. See (3.7)-(3.17) for definitions. Vertical dotted lines delimit the linear and nonlinear phase.

nonlinear phase to start at the same time at both $\operatorname{Pr}$ since the secondary growth rates are different and the nonlinear effects can be felt at different amplitudes at $\operatorname{Pr}=1$ and $\operatorname{Pr}=4$. The linear stage $(t=0$ to $t=400$ for $\operatorname{Pr}=1$ and $t=0$ to $t=150$ for $\operatorname{Pr}=4)$ can be identified as the initial period where the various terms of the budget are time-independent. Figure 8 shows that in the linear stage of the simulations the equality $\sigma_{2}^{K E}=\sigma_{2}^{P E}=\sigma_{2}$ holds true, validating the linear calculations of $\S 3.1$ and the consideration of secondary wave vectors parallel to the $y$-axis only.

In particular, we are looking for indices of a buoyant-convective secondary instability. Buoyant-convective instability is characterized by conversion of potential energy into kinetic energy $\left(X_{3 D}<0\right.$ in (3.7) and (3.14)) since in the limiting case of a purely convective instability there is no mean shear. In practice, both shear production and conversion of potential energy into kinetic energy are important terms in the kinetic energy budget of the secondary instability of stratified $\mathrm{KH}$ billows (Caulfield \& Peltier 2000). However, here for both $\operatorname{Pr}=1$ and $\operatorname{Pr}=4$ the conversion term (circles) is a small term in the kinetic energy budget, production by shear (triangles and squares) being the main source. In the potential energy budget, the conversion term dominates and the production term $\mathscr{H}_{2 D-3 D}$ (squares) is very small. Since the dissipation $\mu_{3 D}$ (crosses) is necessarily positive the main source of potential energy is therefore conversion of kinetic energy into potential energy, and not the converse. Therefore, based on this spatially integrated energy budget, it seems that convective instability plays a minor or negligible role despite the presence of statically unstable regions with a large Rayleigh number.

In order to obtain a more spatialized picture of the energy budget, we now present the spatial distribution of the buoyancy flux $\left\langle w_{2 D} b_{2 D}\right\rangle_{y}$, together with the vertical buoyancy gradient $\sqrt{R i}+\left(\partial b_{2 D} / \partial z\right)$ and the kinetic energy $K E_{3 D}$ (figure 9). Only the 
(a)

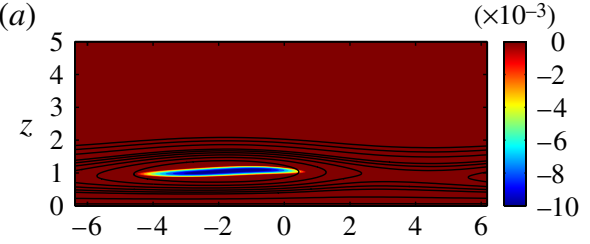

(b)

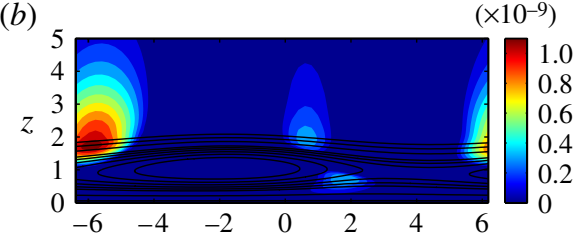

(c)

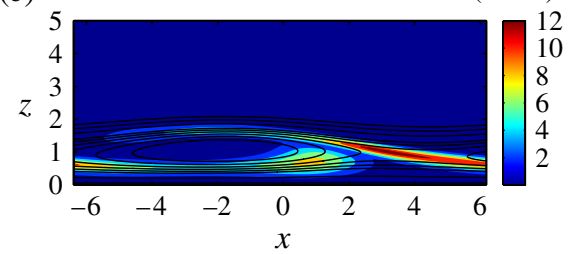

(d)

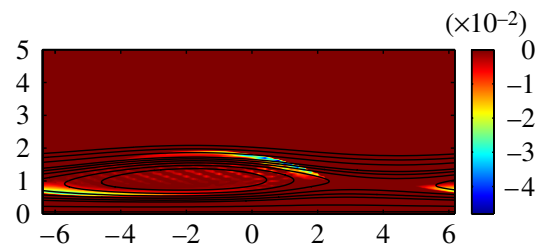

(e)

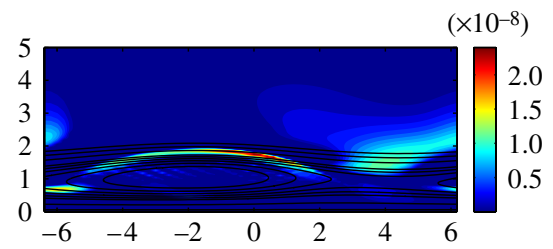

( $f$ )

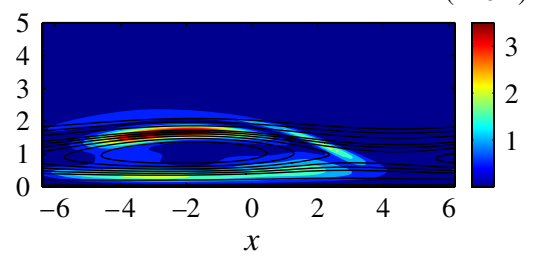

FIGURE 9. Buoyancy gradient $\sqrt{R i}+\partial b_{2 D} / \partial z(a, d)$, buoyancy flux $\left\langle w_{3 D} b_{3 D}\right\rangle_{y}$ (b,e, arbitrary units) and turbulent kinetic energy $K E_{3 D}$ (c,f, arbitrary units) in the linear stage of the development of the secondary instability, for $R e=1000, R i=0.015$ and $\operatorname{Pr}=1(a-c)$, $\operatorname{Pr}=4(d-f)$. Only the negative values of the buoyancy gradient and the buoyancy flux are plotted.

positive values of the buoyancy gradient and the buoyancy flux are plotted, in order to focus on the statically unstable regions and possible local convective instabilities. For $\operatorname{Pr}=1$ the statically unstable region is at the centre of the roll, while the unstable mode develops around the roll. Positive values of the buoyancy flux are found but they do not overlap with the statically unstable region. We conclude that no convective instability is active at $\operatorname{Pr}=1$. This is consistent with the low value of the Rayleigh number $(R a \simeq 10)$. For $\operatorname{Pr}=4$ the statically unstable region consists of two lobes, centred around $x=1, z=1.5$ and $x=-6, z=1$. Now these regions do overlap with regions of positive buoyancy flux, especially at $z>1$ between the seventh and eighth streamline starting from the roll core, suggesting that a convective instability is occurring there. This is consistent with the much larger Rayleigh number $R a \simeq 1400$ found at $R e=1000, \operatorname{Pr}=4, R i=0.015$ (figure 5). However the 3D kinetic energy peaks in a different region, between the third and fourth streamline starting from the roll core. This confirms that, although buoyant-convective instability is occurring locally, it does not contribute significantly to the overall growth of the 3D perturbations.

\subsection{Energetics in the nonlinear stage of secondary instability}

We finally investigate the nonlinear stage of the secondary instability. We focus on a comparison of the two parameter sets $(R e, R i, P r)=(1000,0.015,1)$ and $(R e, R i, P r)=(1000,0.015,4)$. Both flows become very complex after the secondary instability develops and we do not attempt a complete description of their evolution. Instead we focus on a few questions. 

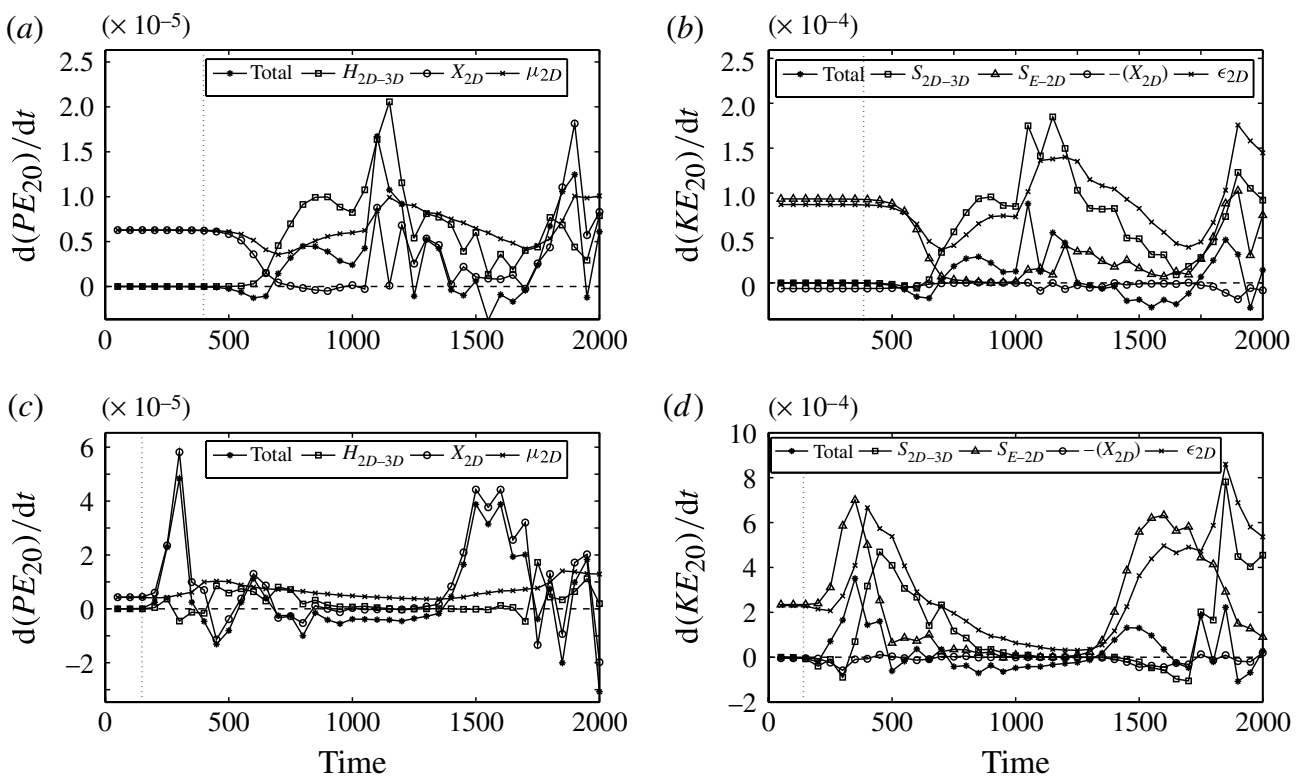

FIGURE 10. Energy budget ( $a, c$ : potential energy; $b, d$ : kinetic energy) of rolls for $R e=1000$ and $R i=0.015$ : $(a, b) \operatorname{Pr}=1 ;(c, d) \operatorname{Pr}=4$. See (3.19)-(3.25) for definitions. Vertical dotted lines delimit the linear and nonlinear phase.

(a) What are the energy exchanges between the 3D fluctuations, the 2D mean flow and the Ekman background flow?

(b) Does the secondary instability lead to the complete breakdown of the initial roll vortex?

(c) If so, does a new vortex re-emerge, subject again to a secondary instability, and giving possibly rise to a cycle of roll vortex emergence/breakdown?

The energetics are analysed using the diagnostics presented in figure 8, complemented by the energy budget of the 2D flow (figure 10) and the temporal evolution of the energies of the 2D and 3D parts of the flow (figure 11). We analyse the breakdown of the roll vortex by additionally inspecting the streamlines of the $y$-averaged (2D) flow (figure 12).

At $\operatorname{Pr}=1$ the energetics of the linear stage of the secondary instability are dominated, as concluded in $\S 3.3$, by shear production of $3 \mathrm{D} K E$. This production is predominantly due to the Ekman background ( $S_{E-3 D}$, figure $8 b$, triangles) and, to a lesser extent, to the $2 \mathrm{D}$ rolls $\left(S_{2 D-3 D}\right.$, figure $8 b$, squares). As long as the $3 \mathrm{D}$ perturbations are small, the rolls remain steady due to a balance between shear production of $\mathrm{KE}$ by the Ekman flow $\left(S_{E-2 D}\right.$, figure $10 b$, triangles) and viscous dissipation $\left(\varepsilon_{2 D}\right.$, figure $10 \mathrm{~b}$, crosses). For $500<t<700$ the $3 \mathrm{D}$ perturbations reach a finite amplitude and modify the $2 \mathrm{D}$ flow in a way that diminishes its ability to extract kinetic energy from the Ekman background $\left(S_{E-2 D}\right.$, figure $10 \mathrm{~b}$, triangles). As a consequence the $2 \mathrm{D} K E$ decays during that interval $\left(K E_{2 D}\right.$, figure $11 a$, squares $)$. For $700<t<1000, K E_{3 D}$ grows and reaches a maximum at $t=1000$. Indeed at this point $3 \mathrm{D}$ viscous dissipation remains significant $\left(\varepsilon_{3 D}\right.$, figure $8 b$, crosses) while $3 \mathrm{D}$ perturbations are no more able to extract $K E$ from the Ekman background and give back KE to the rolls $\left(S_{E-3 D}\right.$ and $S_{2 D-3 D}$, figure $8 b$ ). For $1000<t<2000$ high levels 

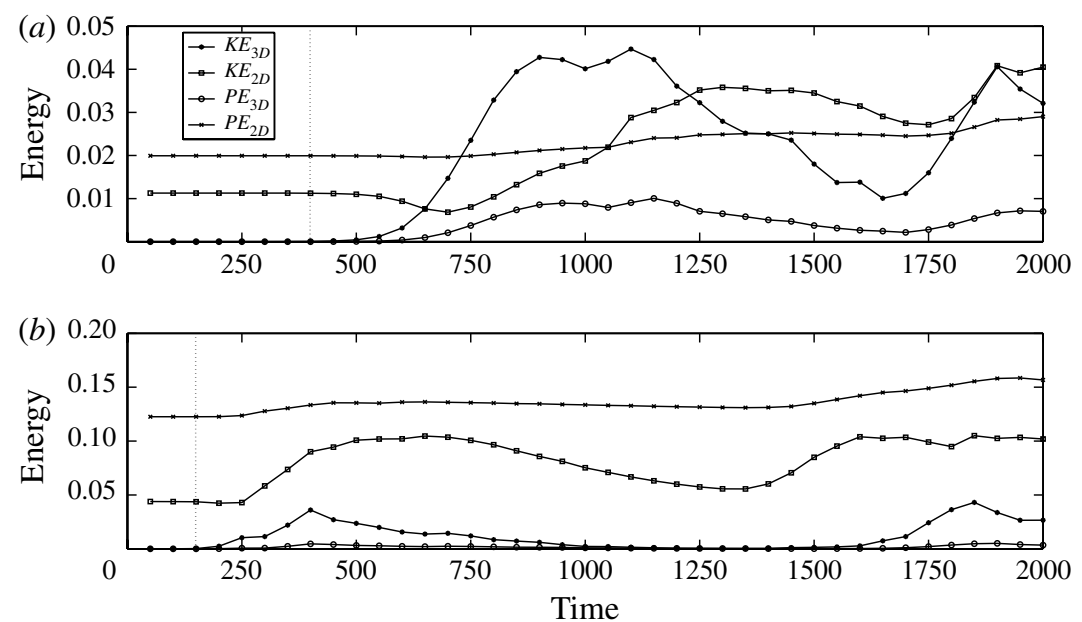

FIGURE 11. Energy of rolls and 3D perturbations for during the development of the secondary instability at $(R e, R i)=(1000,0.015)$ and $\operatorname{Pr}=1(a)$ and $\operatorname{Pr}=4(b)$. Vertical dotted lines delimit the linear and nonlinear phase.
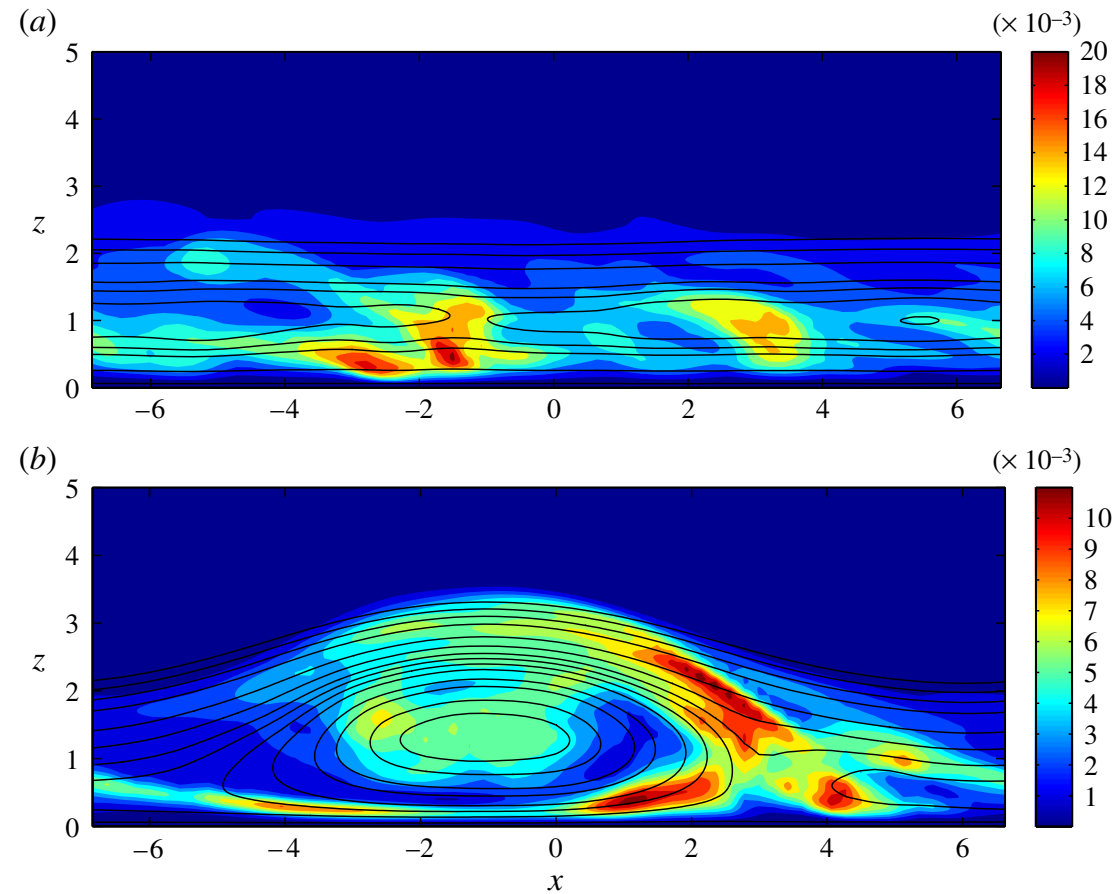

FIGURE 12. Nonlinear development of the secondary instability at $(R e, R i)=(1000,0.015)$ and $\operatorname{Pr}=1(a)$ and $\operatorname{Pr}=4(b)$. Snapshot $(a)$ is taken at $t=1500$ and $(b)$ at $t=1700$. Black curves are streamlines of the $y$-averaged flow. Colours indicate the $y$-averaged turbulent kinetic energy.

of 3D viscous dissipation are reached due to the formation of small-scale features, leading to the decay of the 3D $K E$. During this stage of decay of the 3D perturbations, 
there is still a significant production of $3 \mathrm{D} K E$ by the Ekman shear and transfer of $K E$ from the 3D perturbations to the 2D flow. Regarding the potential energy budget, the only source of $3 \mathrm{D} P E$ is conversion from $3 \mathrm{D} K E$ throughout $\left(X_{3 D}\right.$, figure $8 a, b$, circles). Indeed the interaction between the 3D buoyancy flux and the 2D buoyancy gradient acts as a sink of $3 \mathrm{D} P E\left(\mathscr{H}_{2 D-3 D}\right.$, figure $8 a$, squares) in the nonlinear regime too. After $t=700$ the roll $K E$ grows again due to the transfer of kinetic energy from the 3D perturbations to the 2D flow, complemented after $t=1000$ by extraction of $K E$ from the Ekman background $\left(S_{2 D-3 D}\right.$ and $S_{E-2 D}$, figure $10 b$, squares and triangles). The weak exchange of potential energy between the 3D perturbations and rolls ( $\mathscr{H}_{2 D-3 D}$, figure $10 a$, squares) leads to a weak increase in $2 \mathrm{D} P E\left(P E_{2 D}\right.$, figure $11 a, b$, bold crosses). The roll vortex, identified by closed, elliptically-shaped streamlines is strongly deformed and elongated after the secondary instability develops. A typical snapshot taken at $t=1500$ is presented in figure $12 a$. Three-dimensional perturbations develop again at $t \simeq 1750$ on top of this new background flow which bears little resemblance with the initial roll vortex seen in figure 9. Analysis of the simulation until $t=4000$ (not shown) indicates that there is no cycle of emergence/breakdown of rolls. Instead a relatively constant level of 3D turbulence is reached on top of a $y$-averaged flow resembling that presented in figure 12(a).

At $\operatorname{Pr}=4$, the most important term in the kinetic energy budget of rolls is the term of production ( $S_{E-2 D}$, figure $10 d$, triangles). It is also the most important term of the global budget (figure 10c,d). Rolls extract their kinetic energy mainly from the Ekman flow for $0<t<150$ ( $S_{E-2 D}$, figure $10 d$, triangles). We note also that the conversion of $2 \mathrm{D}$ kinetic energy into $2 \mathrm{D}$ potential energy dominates the potential energy budget ( $X_{2 D}$, figure $10 c, d$, circles). As for $P r=1, K E_{3 D}$ grows and reaches a maximum but for a shorter time interval $150<t<400$. This phase of growth of the 3D perturbations is followed by a decay phase for $400<t<1100\left(K E_{3 D}\right.$, figure $11 b$, stars) during which rolls do not extract their energy from Ekman flow but from 3D perturbations $\left(S_{2 D-3 D}\right.$, figure $10 d$, squares). The source of 3D perturbations energy remains the shear production by Ekman of $K E_{3 D}\left(S_{E-3 D}\right.$, figure $8 d$, triangles) while conversion of $K E_{3 D}$ into $P E_{3 D}$ ( $X_{3 D}$, figure $10 d$, circles) acts consistently as a sink of $K E_{3 D}$ albeit of negligible magnitude. In this phase $K E_{3 D}$ decreases due to the important dissipation which reaches the same order of magnitude as shear production by the Ekman flow and exceeds it ( $\epsilon_{3 D}$ and $S_{E-3 D}$, figure $8 d$, crosses and triangles). Another cause is that $3 \mathrm{D}$ perturbations lose kinetic energy through transfer in favour of rolls $\left(S_{2 D-3 D}\right.$, figure $10 d$, squares). At $t=1100$, although $K E_{2 D}$ is at a higher level than at the beginning of the simulation, the $y$-mean flow has lost its roll structure since its streamlines are all open (not shown). The $y$-mean flow remains quasi-parallel until $t \simeq 1350$ then a new 2D roll grows for $1350<t<1700$. Meanwhile a secondary instability grows on top of this new roll flow. Figure 12(b) displays at $t=1700$ the streamlines of the $y$-mean flow which have a well-defined roll pattern as well as the $y$-averaged kinetic energy of 3D perturbations (colour) that have developed. Again the nonlinear development of this $3 \mathrm{D}$ instability destroys the roll pattern at $t \simeq 1900$. Analysis of the simulation until $t=4000$ (not shown) indicates (as for $P r=1$ ) that there is no cycle of emergence/breakdown of rolls.

\section{Conclusions}

We have studied the effect of stratification on saturated rolls emerging from the linear instability of a stratified Ekman flow. The effect of stratification, measured by the bulk Richardson number $R i$, depends strongly on the Prandtl number. For $\operatorname{Pr}=1$, 
the potential energy of saturated rolls grows with $R i$ for weak stratifications, reaches a maximum then decreases until it vanishes for $R i$ close to the critical Richardson number. The initial growth of the potential energy for the low values of $R i$ can be understood by the fact that rolls remain rather strong and can extract potential energy from the stratified environment by lifting heavy fluid. When the stratification increases, rolls weaken substantially and become unable to lift heavy fluid, and their energy (kinetic and potential) eventually vanishes. A different evolution as $R i$ increases is observed for $\operatorname{Pr}=4$. Indeed the potential energy increases steadily as $R i$ increases, and remains finite when $R i$ reaches $R i_{c}$. Hence, the bifurcation at $R i=R i_{c}$ is subcritical for $\operatorname{Pr}=4$ while it is supercritical for $\operatorname{Pr}=1$. Previous work on the Ekman layer stability diagram has characterized the bifurcation in the non-stratified situation only. Haeusser \& Leibovich (2003) and Dubos et al. (2008) find a supercritical bifurcation when the Reynolds number reaches its critical value. A detailed investigation of the change from a supercritical bifurcation to a subcritical bifurcation as $\operatorname{Pr}$ is increased is under way. Preliminary results suggest that this Prandtl number effect is quite generic and exists for general stratified shear flows.

Brown (1972) speculates, based on inspection of the (presumably nonlinear) Boussinesq equations, that the dynamics of the stratified Ekman layer depend weakly on $R a, R e, P r$. This suggestion is confirmed by his results which deal with the primary linear stability analysis: for sufficiently large $R e$ the growth rate depends weakly on $\operatorname{Re}, \operatorname{Pr}$ and the critical Richardson number depends weakly on $R e$ and almost not at all on $P r$. We reproduce these results in $\S 2$. However, Brown's speculation fails when nonlinear effects come into play, as demonstrated by the strong dependence of the saturation amplitude of the rolls on $\mathrm{Pr}$.

We have evaluated the a priori potential for the Ekman roll flow to develop a secondary convective instability. Like the $\mathrm{KH}$ billows, the rolls present statically unstable regions associated to large local Rayleigh numbers, especially at large $R e$ and Pr. Again a strong Prandtl number effect is found. In terms of Rayleigh number, rolls forming at $\operatorname{Pr}=1$ do not create conditions very favourable to convective instability, even at a relatively large Reynolds number $\operatorname{Re}=1000$. At $\operatorname{Pr}=4$ the Rayleigh number is much larger, above typical values of the critical Rayleigh number. Nevertheless the contribution of convective instability to the secondary instability is minor, as shown by our secondary stability study. Whether a convective instability effectively develops was a central question in our subsequent numerical investigation of the secondary instability.

Linear secondary stability analysis shows that qualitatively different branches are selected depending on $R i$. For small enough $R i$ the fastest growing secondary mode has a relatively large wavenumber and belongs to the same instability branch as when $R i=0$. A qualitative effect of $R i$ is found at higher values. There the fastest growing modes have a much lower wavenumber $k_{y}<1$. This is at stark contrast with the secondary instability of $\mathrm{KH}$ billows, for which the fastest growing mode has a large wavenumber, which furthermore grows as $R i$ is increased. The Prandtl number affects mainly how the secondary growth rate $\sigma_{2}$ evolves as $R i$ is increased. For $\operatorname{Pr}=1, \sigma_{2}$ decreases as $R i$ increases and vanishes at $R i=R i_{c}$ while it remains significant for $\operatorname{Pr}=4$ over the range of stratification studied. This behaviour is likely a result of the roll vortices weakening as $R i$ approaches its critical value if $\operatorname{Pr}=1$, but not if $\operatorname{Pr}=4$.

The energetics of the linear secondary instability were detailed in order to assess the presence of a convective instability. Spatially averaged energy budgets show that convection plays a negligible role in helping the 3D perturbations to extract energy from the base flow. The main source of energy is the production by shear. In fact 
the conversion between potential and kinetic energy of the $3 \mathrm{D}$ perturbations is at the expense of kinetic energy. This conversion is the only net source of 3D potential energy. This result is nuanced by a more local examination of the energy budget. At $P r=4$ statically unstable regions of the base flow coincide with a positive buoyancy flux, suggesting the occurrence of a convective instability at least locally. Given the minor role of convective instability at $\operatorname{Pr}=1$ we have not studied $\operatorname{Pr}<1$, especially the atmospheric value $P r=0.7$. Indeed lowering $P r$ reduces the buoyancy gradients and the local Rayleigh number, making the occurrence of convective instability even less probable (figure 5). Note that a similar Prandtl-number dependence of the Rayleigh number has been found in KH billows (Mashayek \& Peltier 2012).

For seawater, the (thermal) Prandtl number is $\sim 7$ and the (haline) Schmidt number is $\sim 700$, much higher than what we could achieve for this study. Both are significantly larger than one, and the finding obtained in $\S 3$ that the bifurcation at the critical Richardson number is subcritical applies. Note that a more systematic investigation of this bifurcation confirms that its subcritical/supercritical character is dominantly controlled by the Prandtl number, and that the underlying mechanism applies generally to stratified shear floes, including the continuously stratified KH flow (Mkhinini, Dubos \& Drobinski 2013). At near-critical Richardson numbers this should favour the occurrence of turbulence in the ocean, compared to atmospheric flows for which $\operatorname{Pr}=0.7$. Furthermore the Schmidt number for salt is so high that the convective secondary instability, which is weak and subdominant at $\operatorname{Pr}=4$, may be much more active. To establish this, both transition to turbulence and the fully turbulent regime of the stratified Ekman layer should be investigated for really high values of $\operatorname{Pr}$, maybe in experiments if it is numerically too challenging to reach high $R e$ and $P r$ simultaneously.

Why the role of the convective instability is so much less important for the secondary instability of the Ekman rolls than for the secondary instability of $\mathrm{KH}$ billows is not obvious. Apart from the presence of a solid wall, the most obvious difference between the two base flows is the presence of an along-roll (spanwise) flow due to the veering of the Ekman laminar flow caused by the Coriolis force. This spanwise flow, which possesses its own shear, may adversely affect the development of the convective instability. Indeed the secondary instability of $\mathrm{KH}$ billows leads to the development of intense spanwise-periodic streamwise vortices (Peltier \& Caulfield 2003) while sheared convection tends to develop rolls whose axes are aligned with the shear. Stratified shear turbulence is a topic of active current research. These results suggest that more attention should be paid to the situation where the mean shear is not unidirectional, whatever the cause for the veering of the horizontal-mean flow.

\section{Acknowledgements}

We are grateful to C.-C. Caulfield for fruitful discussions and suggestions. This work was supported by the INSU/LEFE program and by the Agence Nationale de la Recherche, contract ANR-09-JCJC-0108-01. Numerical calculations were performed at the IDRIS computing facility as part of project x2011016033.

\section{Appendix. Numerical methods}

\section{A.1. Spatial discretization}

In all circumstances we actually solve for the deviations $\boldsymbol{U}$ from the Ekman profile and $b$ from the linear background stratification. The vertical coordinate $z$ goes from 0 at the ground to $+\infty$ and is mapped to $\eta=1-2 \mathrm{e}^{-z / z_{0}} \in[-1,1]$. This interval is equally 
divided into $N_{z}$ elements, in each of which we define six Gauss-Legendre quadrature points. The free parameter $z_{0}$ controls the thickness of the well-resolved region above $z=0$ and is set to $z_{0}=4.1$ in the present work. We also we define $N_{z}+4$ cubic B-splines $S_{m}(\eta)$ satisfying

$$
\left.\frac{\mathrm{d} S_{m}}{\mathrm{~d} \eta}\right|_{\eta=-1}=0, \quad m \geqslant 1,\left.\quad \frac{\mathrm{d}^{2} S_{m}}{\mathrm{~d} \eta^{2}}\right|_{\eta=-1}=0, \quad m \geqslant 2 .
$$

The basis functions $S_{m}(\eta)$ are evaluated at the $6 N_{z}$ quadrature points $\eta_{n}$. Cubic Bsplines span four elements, hence the resulting $6 N_{z} \times\left(N_{z}+3\right)$ matrix $\boldsymbol{S}=\left(S_{m}\left(\eta_{n}\right)\right)$ is sparse with $\sim 24 N_{z}$ non-zero entries. We note $\boldsymbol{S}_{1}$ the same matrix with the first column omitted and $\boldsymbol{S}_{2}$ the same matrix with the first two columns omitted. Within each element, polynomials of degree up to five can be differentiated exactly through Lagrange interpolation at quadrature points. We note $\mathrm{d} / \mathrm{d} \eta$ the corresponding matrix made of $N_{z}$ diagonal blocks of size $6 \times 6$ and $\mathrm{d} \eta / \mathrm{d} z$ the diagonal matrix with entries $2\left(1-\eta_{n}\right) / z_{0}$. Vertical differentiation is performed by left-multiplication by

$$
\boldsymbol{G}=\frac{\mathrm{d} \eta}{\mathrm{d} z} \cdot \frac{\mathrm{d}}{\mathrm{d} \eta} .
$$

We work with finite-dimensional spaces for velocity and buoyancy satisfying horizontally periodic boundary conditions, Dirichlet boundary conditions at $z=0$ and exponential decay as $z \rightarrow \infty$. Multiplying $S_{m}(\eta)$ by $1-\eta=2 \mathrm{e}^{-z / z}$ ensures exponential decay for $z \rightarrow \infty$ while the Dirichlet boundary condition at $z=0$ is obtained by excluding the first or first two B-splines from the expansions. For velocity we use a toroidal-poloidal decomposition to ensure non-divergence and treat the horizontalmean flow separately:

$$
\begin{aligned}
b(x, y, z, t)= & \sum_{k=0}^{M_{x}} \sum_{l=-M_{y}}^{M_{y}} \sum_{m=1}^{M_{z}} \tilde{b}_{k l m} \operatorname{expi}\left(\frac{k x}{2 \pi L_{x}}+\frac{l y}{2 \pi L_{y}}\right)(1-\eta) S_{m}(\eta)+\text { c.c. } \\
\alpha(x, y, z)= & \sum_{k=0}^{M_{x}} \sum_{l=-M_{y}}^{M_{y}} \sum_{m=1}^{M_{z}} \tilde{\alpha}_{k l m} \operatorname{expi}\left(\frac{k x}{2 \pi L_{x}}+\frac{l y}{2 \pi L_{y}}\right)(1-\eta) S_{m}(\eta)+\text { c.c. } \\
\beta(x, y, z)= & \sum_{k=0}^{M_{x}} \sum_{l=-M_{y}}^{M_{y}} \sum_{m=2}^{M_{z}} \tilde{\beta}_{k l m} \operatorname{expi}\left(\frac{k x}{2 \pi L_{x}}+\frac{l y}{2 \pi L_{y}}\right)(1-\eta) S_{m}(\eta)+\text { c.c. } \\
\boldsymbol{U}(x, y, z)= & U(z) \boldsymbol{e}_{x}+V(z) \boldsymbol{e}_{y}+\nabla \times\left(\alpha(x, y, z) \boldsymbol{e}_{z}+\nabla \times\left(\beta(x, y, z) \boldsymbol{e}_{z}\right)\right) \\
= & \sum_{m=1}^{M_{z}}\left(U_{m}(1-\eta) S_{m}(\eta) \boldsymbol{e}_{x}+V_{m} S_{m}(\eta) \boldsymbol{e}_{y}\right) \\
& +\sum_{k=0}^{M_{x}} \sum_{l=-M_{y}}^{M_{y}} \sum_{m=0}^{M_{z}} \tilde{\alpha}_{k l m} \operatorname{expi}\left(\frac{k x}{2 \pi L_{x}}+\frac{l y}{2 \pi L_{y}}\right) \boldsymbol{U}_{k l m}^{\text {toro }}(\eta)+\text { c.c. } \\
& +\sum_{k=0}^{M_{x}} \sum_{l=-M_{y}}^{M_{y}} \sum_{m=0}^{M_{z}} \tilde{\beta}_{k l m} \operatorname{expi}\left(\frac{k x}{2 \pi L_{x}}+\frac{l y}{2 \pi L_{y}}\right) \boldsymbol{U}_{k l m}^{p o l o}(\eta)+\text { c.c. }
\end{aligned}
$$


This method is a modification of the spectrally accurate spatial discretization described in more detail by Spalart (1989) and used in earlier studies of the Ekman boundary layer (Coleman, Ferziger \& Spalart 1990; Foster 1996; Dubos et al. 2008). The main modification is the replacement of the Jacobi polynomials by cubic B-splines to improve efficiency at the price of fourth-order, instead of spectral, accuracy. The discrete degrees of freedom are the real coefficients $U_{m}, V_{m}$ and the complex coefficients $\tilde{\alpha}_{k l m}, \tilde{\beta}_{k l m}, \tilde{b}_{k l m}$. In the following the column-vector obtained by concatenating all of these coefficients is denoted by $\tilde{\boldsymbol{q}}$, while the continuous flow state $(\boldsymbol{u}, b)$ is denoted by $\boldsymbol{q}$. The coefficients corresponding to Fourier mode $k, l$ are denoted by $\tilde{\boldsymbol{q}}_{k l}$.

Discrete problems are obtained from the continuous problems by Galerkin projection onto the spaces defined above. Since we work with non-divergent flow fields, the pressure gradient disappears after Galerkin projection and we omit it when writing the continuous problems.

\section{A.2. Primary linear stability}

For primary linear stability $L_{x}=2 \pi / k_{1}$, the expansions (A 3)-(A 9) are truncated to $M_{x}=1, M_{y}=0$ and the mean flow is not involved. Given the column-vector $\tilde{\boldsymbol{b}}=\left(\tilde{b}_{m}\right)_{m \geqslant 1}$, the values $b\left(z_{n}\right)$ are obtained as $(1-\eta) \boldsymbol{S}_{1} \cdot \tilde{\boldsymbol{b}}$ where $\boldsymbol{B}=(1-\eta) \cdot \boldsymbol{S}_{1}$ and $(1-\eta)$ is shorthand notation for the diagonal matrix with entries $1-\eta_{n}$. Matrices similar to $\boldsymbol{B}$ are defined for the toroidal and poloidal components of velocity, i.e.

$$
\boldsymbol{T}=\left[\begin{array}{c}
0 \\
\mathrm{i} k_{1} \boldsymbol{S}_{1} \\
0
\end{array}\right], \quad \boldsymbol{P}=\left[\begin{array}{c}
-\mathrm{i} k_{1} \boldsymbol{G} \cdot \boldsymbol{S}_{2} \\
0 \\
-k_{1}^{2} \boldsymbol{s}_{2}
\end{array}\right]
$$

which shows that the toroidal-poloidal decomposition is equivalent in $2 \mathrm{D}$ to a decomposition into $y$-component and streamfunction. Given the complex columnvector $\tilde{\boldsymbol{q}}=\left(\tilde{\alpha}_{m}, \tilde{\beta}_{m}, \tilde{b}_{m}\right)$ (we omit indices $k=1, \quad l=0$ ), the values of $u, v, w, b$ corresponding to expansions (A 3)-(A 9) are obtained at quadrature points as

$$
\left[\begin{array}{c}
u_{n} \\
v_{n} \\
w_{n} \\
b_{n}
\end{array}\right]=\boldsymbol{Q} \cdot \tilde{\boldsymbol{q}} \quad \text { where } \boldsymbol{Q}=\left[\begin{array}{ccc}
\boldsymbol{T} & \boldsymbol{P} & 0 \\
0 & 0 & \boldsymbol{B}
\end{array}\right]
$$

Corresponding to the continuous linearized operator

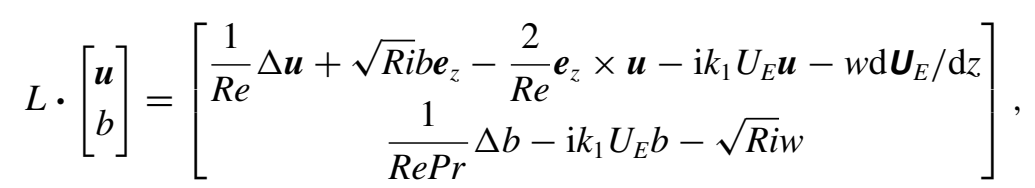

we define the $24 N_{z} \times 24 N_{z}$ block-structured sparse matrix

$$
L=\left[\begin{array}{cccc}
\frac{1}{R e}\left(\boldsymbol{G} \cdot \boldsymbol{G}-k_{1}^{2}\right)-\mathrm{i} k_{1} U_{E} & \frac{2}{R e} \mathbf{1} & -U_{E}^{\prime} & \\
-\frac{2}{R e} \mathbf{1} & \frac{1}{R e}\left(\boldsymbol{G} \cdot \boldsymbol{G}-k_{1}^{2}\right)-\mathrm{i} k_{1} U_{E} & -V_{E}^{\prime} & \\
& & \frac{1}{R e}\left(\boldsymbol{G} \cdot \boldsymbol{G}-k_{1}^{2}\right)-\mathrm{i} k_{1} U_{E} & -\sqrt{R i} \mathbf{1} \\
& & \sqrt{R i} \mathbf{1} & \frac{1}{\operatorname{RePr}}\left(\boldsymbol{G} \cdot \boldsymbol{G}-k_{1}^{2}\right)-\mathrm{i} k_{1} U_{E}
\end{array}\right]
$$


where $U_{E}, U_{E}^{\prime}, V_{E}^{\prime}$ are diagonal matrices. The discrete eigenvalue problem for the vector $\tilde{\boldsymbol{q}}$ is set up via a Galerkin projection. For this we use the scalar product

$$
\begin{aligned}
& \left\langle\boldsymbol{q}, \boldsymbol{q}^{\prime}\right\rangle=\int\left(\boldsymbol{u}^{*} \cdot \boldsymbol{u}^{\prime}+b^{*} b^{\prime}\right) \mathrm{d} z=\sum_{n} \mu_{n}\left(u_{n}^{*} u_{n}^{\prime}+v_{n}^{*} v_{n}^{\prime}+w_{n}^{*} w_{n}^{\prime}+b_{n}^{*} b_{n}^{\prime}\right) \\
& \left\langle\tilde{\boldsymbol{q}}, \tilde{\boldsymbol{q}}^{\prime}\right\rangle=\tilde{\boldsymbol{q}}^{*} \cdot \boldsymbol{M} \cdot \tilde{\boldsymbol{q}}, \quad \boldsymbol{M}=\boldsymbol{Q}^{*} \cdot \boldsymbol{\mu} \cdot \boldsymbol{Q}
\end{aligned}
$$

where $*$ is the conjugate-transpose, $\mu_{n}$ are Gauss-Legendre weights divided by the mapping factor $\mathrm{d} \eta / \mathrm{d} z=(1-\eta) / z_{0}$ and $\boldsymbol{\mu}$ is the diagonal matrix with values $\mu_{n}$. Note that the factor $(1-\eta)$ used in (A 3)-(A 9) to define the basis functions avoids any singularity in the above integral and quadrature formula. The mass matrix $\boldsymbol{M}$ is symmetric positive-definite and sparse with a banded structure. Upon Galerkin projection we obtain a generalized eigenproblem

$$
\left(\boldsymbol{R}-\sigma_{1} \boldsymbol{M}\right) \cdot \tilde{\boldsymbol{q}}=0, \quad \boldsymbol{R}=\boldsymbol{Q}^{*} \cdot \boldsymbol{\mu} \cdot \boldsymbol{L} \cdot \boldsymbol{Q}
$$

where the stiffness matrix $\boldsymbol{R}$ is also sparse and banded. This eigenproblem is solved for $\sigma_{1}$ and $\tilde{\boldsymbol{q}}$ by a direct $\mathrm{QR}$ method providing all discrete eigenvalues. All calculations are done with Matlab.

\section{A.3. Three-dimensional initial-value problems}

Now the full expansions (A 3)-(A 9) are used. They define finite-dimensional spaces $\mathscr{V}, \mathscr{B}$ and $\mathscr{Q}=\mathscr{V} \oplus \mathscr{B}$ for velocity, buoyancy and the complete flow state $\boldsymbol{q}=(\boldsymbol{u}, b)$. The Boussinesq equations can be written symbolically as

$$
\frac{\partial \boldsymbol{q}}{\partial t}=\Delta \boldsymbol{q}+\boldsymbol{a}(\boldsymbol{q})
$$

where $\Delta \boldsymbol{q}$ gathers the viscous and diffusive terms and $\boldsymbol{a}(\boldsymbol{q})$ gathers the other, conservative terms. In general $\boldsymbol{a}(\boldsymbol{q})+\Delta \boldsymbol{q}$ is not in $\mathscr{Q}$ when $\boldsymbol{q} \in \mathscr{Q}$. Therefore, when performing temporal integrations, we solve (A 17) in the Galerkin sense, e.g. we search $\boldsymbol{q}(t)$ in $\mathscr{Q}$ such that

$$
\forall \hat{\boldsymbol{q}} \in \mathscr{Q} \quad\left\langle\hat{\boldsymbol{q}}, \partial_{t} \boldsymbol{q}\right\rangle=\langle\hat{\boldsymbol{q}}, \Delta \boldsymbol{q}\rangle+\langle\hat{\boldsymbol{q}}, \boldsymbol{a}(\boldsymbol{q})\rangle
$$

where $\hat{\boldsymbol{q}} \in \mathscr{Q}$ is an arbitrary test field. Scalar products are computed as a weighted sum of values at quadrature points $\left(x_{i}, y_{j}, \eta_{n}\right)$ :

$$
\left\langle\boldsymbol{q}, \boldsymbol{q}^{\prime}\right\rangle=\int\left(\boldsymbol{u} \cdot \boldsymbol{u}^{\prime}+b b^{\prime}\right) \mathrm{d} x \mathrm{dy} \mathrm{d} z=\sum_{i j n} \mu_{n}\left(u_{i j n} u_{i j n}^{\prime}+v_{i j n} v_{i j n}^{\prime}+w_{i j n} w_{i j n}^{\prime}+b_{i j n} b_{i j n}^{\prime}\right)(
$$

where $u_{i j n}=u\left(x_{i}, y_{j}, z_{n}\right)$, etc. We use $N_{x} \times N_{y}$ equally spaced points $x_{i}, y_{j}$ and the 2/3 dealiasing rule $M_{x} \leqslant N_{x} / 3, M_{y} \leqslant N_{y} / 3$. Expansions (A 3)-(A 9) define mappings $\tilde{\boldsymbol{q}} \mapsto \boldsymbol{u}, \boldsymbol{\omega}=$ curl $\boldsymbol{u}, b, \boldsymbol{\nabla} b$. In practice computing $\boldsymbol{u}, \boldsymbol{\omega}, b, \boldsymbol{\nabla} b$ at quadrature points from $\tilde{\boldsymbol{q}}$ combines horizontal fast Fourier transforms and several applications of the matrices $\boldsymbol{S}_{1}, \boldsymbol{S}_{2}, \boldsymbol{G}$. Then $\boldsymbol{a}(\boldsymbol{q})$ can be computed independently at each quadrature point. Finally to compute the dot products of $\boldsymbol{a}(\boldsymbol{q})$ against all test functions one multiplies $\boldsymbol{a}(\boldsymbol{q})$ by the weights $\mu_{n}$, and applies the transposed matrices $\boldsymbol{S}_{1}^{*}, \boldsymbol{S}_{2}^{*}, \boldsymbol{G}^{*}$ and horizontal fast Fourier transforms. We denote the final result by $\tilde{\boldsymbol{a}}(\tilde{\boldsymbol{q}})$. 
The dissipative terms are written as

$$
\begin{aligned}
\langle\hat{\boldsymbol{q}}, \Delta \boldsymbol{q}\rangle & =-\frac{1}{R e}\langle\operatorname{curl} \hat{\boldsymbol{u}}, \operatorname{curl} \boldsymbol{u}\rangle-\frac{1}{\operatorname{RePr}}\langle\nabla \hat{b}, \nabla b\rangle \\
& =-\sum_{k l} \hat{\boldsymbol{q}}_{k l}^{*} \cdot \boldsymbol{D}_{k l} \cdot \tilde{\boldsymbol{q}}_{k l}
\end{aligned}
$$

In this computation the Fourier modes are decoupled and a stiffness matrix $\boldsymbol{D}_{k l}$ is defined for each mode. Similarly a mass matrix $\boldsymbol{M}_{k l}$ is defined for each Fourier mode such that $\langle\hat{\boldsymbol{q}}, \boldsymbol{q}\rangle=\sum_{k l} \hat{\boldsymbol{q}}_{k l}^{*} \cdot \boldsymbol{M}_{k l} \cdot \tilde{\boldsymbol{q}}_{k l}$. The expressions of $\boldsymbol{D}_{k l}$ and $\boldsymbol{M}_{k l}$ follow from expansions (A 3)-(A 9) and involve $\boldsymbol{S}_{1}, \boldsymbol{S}_{2}, \boldsymbol{G}, \boldsymbol{\mu}$ and their transpose. Matrices $\boldsymbol{M}_{k l}$ and $\boldsymbol{D}_{k l}$ are banded, symmetric, positive. Putting all pieces together one obtains a system of ordinary differential equations for vector $\tilde{\boldsymbol{q}}$ :

$$
\forall k l, \quad \boldsymbol{M}_{k l} \cdot \frac{\partial \tilde{\boldsymbol{q}}_{k l}}{\partial} t+\boldsymbol{D}_{k l} \cdot \tilde{\boldsymbol{q}}_{k l}=\tilde{\boldsymbol{a}}_{k l}(\tilde{\boldsymbol{q}})
$$

In (A 21) the coefficients $\tilde{\boldsymbol{a}}(\tilde{\boldsymbol{q}})$ are split into blocks $\tilde{\boldsymbol{a}}_{k l}(\tilde{\boldsymbol{q}})$ corresponding to each Fourier mode but in practice are computed all at once for efficiency. System (A 21) of ordinary differential equations is marched forward in time using a third-order semi-implicit Runge-Kutta scheme. The implicit part deals with dissipative terms with unconditional stability and involves the inversion of matrices of the form $\boldsymbol{M}_{k l}+\tau \boldsymbol{D}_{k l}$, which are symmetric positive-definite and banded. The explicit part is subject to a Courant-Friedrichs-Lewy stability condition.

\section{A.4. Krylov post-processing}

To determine the linear growth rate of the secondary instability, we perform 3D temporal simulations of the Boussinesq equations linearized about the saturated Ekman rolls, in the translating reference frame where the latter are steady. The domain dimension $L_{y}$ and resolution $N_{y}$ along the $y$-axis determine the wavenumbers $k_{y}=2 \mathrm{i} \pi l y / L_{y}\left(l=1 \ldots N_{y} / 2\right)$ for which the secondary growth rate is computed. The initial condition is a random perturbation $\tilde{\boldsymbol{q}}^{0}$, and snapshots $\tilde{\boldsymbol{q}}^{n}$ at regular intervals $t=n T, n \leqslant N$, are taken. Denoting by $\tilde{\boldsymbol{q}}_{l}$ all coefficients describing the Fourier mode $\exp 2 \mathrm{i} \pi l y / L_{y}$, we have $\tilde{\boldsymbol{q}}_{l}^{n}=\left(L_{l}\right)^{n} \cdot \tilde{\boldsymbol{q}}_{l}^{0}$ where $L_{l}$ is the linearized operator acting on the space of modes $\sim \exp 2 \mathrm{i} \pi l y / L_{y}$. The basic idea of the method is to perform a Galerkin projection onto the Krylov spaces $K_{l}=\operatorname{span}\left\{\tilde{\boldsymbol{q}}_{l}^{0}, \ldots \tilde{\boldsymbol{q}}_{l}^{N-1}\right\}$. In practice, each basis $\tilde{\boldsymbol{q}}_{l}^{0}, \ldots \tilde{\boldsymbol{q}}_{l}^{N-1}$ is first orthogonalized with respect to energy to produce an orthonormal basis $\tilde{\boldsymbol{Q}}_{l}^{0}, \ldots \tilde{\boldsymbol{Q}}_{l}^{N-1}$. This process produces coefficients $\alpha_{l}^{m n}$ such that $\tilde{\boldsymbol{q}}_{l}^{m}=\sum_{n \leqslant m} \alpha_{l}^{m n} \tilde{\boldsymbol{Q}}_{l}^{m}$. Small approximate eigenproblems $\left(L_{l}-\sigma_{l}\right) \cdot \tilde{\boldsymbol{q}}_{l} \perp K_{l}$ with $\tilde{\boldsymbol{q}}_{l}=\sum_{m} \beta_{l}^{m} \tilde{\boldsymbol{q}}_{l}^{m}$ are then solved:

$$
\forall n<N, \quad\left\langle\tilde{\boldsymbol{Q}}_{l}^{n}, L_{l} \cdot \sum_{m} \beta_{l}^{m} \tilde{\boldsymbol{q}}_{l}^{m}\right\rangle=\sigma_{l}\left\langle\tilde{\boldsymbol{Q}}_{l}^{n}, \sum_{m} \beta_{l}^{m} \tilde{\boldsymbol{q}}_{l}^{m}\right\rangle .
$$

Since $L_{l} \cdot \tilde{\boldsymbol{q}}_{l}^{m}=\tilde{\boldsymbol{q}}_{l}^{m+1}$, all coefficients involved in the approximate eigenproblems are given by $\alpha_{l}^{m n}=\left\langle\tilde{\boldsymbol{Q}}_{l}^{n}, \tilde{\boldsymbol{q}}_{l}^{m}\right\rangle$. The projection error is estimated by the residual $r_{l}$ of the projection of $\tilde{\boldsymbol{q}}_{l}^{N}$ onto $K_{l}$. If $r_{l} \geqslant \varepsilon \tilde{\boldsymbol{q}}_{l}^{N}$, where $\varepsilon$ is a prescribed tolerance, the eigenvalue/eigenmode is deemed unreliable and ignored. After some trial/error one finds $T$ sufficiently large (here we use $T=50$ ) for the tolerance criterion to be satisfied for $N=10$. 


\section{A.5. Two-dimensional travelling rolls}

For this computation we work with expansions (A 3)-(A 9) truncated to $l=0$ $(\partial / \partial y=0)$. In the reference frame where the solid boundary is steady, the problem we wish to solve is

$$
\forall \hat{\boldsymbol{q}} \in \mathscr{Q} \quad-c\left\langle\hat{\boldsymbol{q}}, \partial_{x} \boldsymbol{q}\right\rangle=\langle\hat{\boldsymbol{q}}, \Delta \boldsymbol{q}\rangle+\langle\hat{\boldsymbol{q}}, \boldsymbol{a}(\boldsymbol{q})\rangle
$$

where the phase velocity and the coefficients $\tilde{\boldsymbol{q}}$ are unknown. We first eliminate the nonlinear phase velocity, making it a dependant quantity instead of an additional unknown. For this, we define $c(\boldsymbol{q})$ by

$$
c(\boldsymbol{q})=-\frac{\left\langle\partial_{x} \boldsymbol{q}, \Delta \boldsymbol{q}\right\rangle+\left\langle\partial_{x} \boldsymbol{q}, \boldsymbol{a}(\boldsymbol{q})\right\rangle}{\left\langle\partial_{x} \boldsymbol{q}, \partial_{x} \boldsymbol{q}\right\rangle} .
$$

Now we are left with a nonlinear problem:

$$
\forall \hat{\boldsymbol{q}} \in \mathscr{Q}, \quad 0=\langle\hat{\boldsymbol{q}}, \Delta \boldsymbol{q}\rangle+\langle\hat{\boldsymbol{q}}, \boldsymbol{a}(\boldsymbol{q})\rangle+\left\langle\hat{\boldsymbol{q}}, c(\boldsymbol{q}) \partial_{x} \boldsymbol{q}\right\rangle,
$$

of the form $\tilde{\boldsymbol{r}}(\tilde{\boldsymbol{q}})=0$, where the only unknown is $\tilde{\boldsymbol{q}}$. Computing $\tilde{\boldsymbol{r}}(\tilde{\boldsymbol{q}})$ and computing the trends for the temporal simulations is the same, except for the additional term due to the phase velocity $c$.

We solve (A 25) by a Newton-Krylov method as follows. Given an initial guess $\tilde{\boldsymbol{q}}_{0}$ we compute a sequence $\left(\tilde{\boldsymbol{q}}_{0}, \tilde{\boldsymbol{q}}_{1}, \ldots\right)$ and two auxiliary sequences $\left(\tilde{\boldsymbol{r}}_{0}, \tilde{\boldsymbol{r}}_{0}, \ldots\right)$ and $\left(\delta \tilde{\boldsymbol{q}}_{0}, \delta \tilde{\boldsymbol{q}}_{1}, \ldots\right)$ as

$$
\begin{aligned}
\boldsymbol{L}_{n} \cdot \delta \tilde{\boldsymbol{q}}_{n} & =\tilde{\boldsymbol{r}}_{n} \\
\tilde{\boldsymbol{q}}_{n+1} & =\tilde{\boldsymbol{q}}_{n}-\delta \tilde{\boldsymbol{q}}_{n}
\end{aligned}
$$

where $\boldsymbol{L}_{n}$ is the linearization of $\tilde{\boldsymbol{r}}$ around $\tilde{\boldsymbol{q}}_{n}$ :

$$
\tilde{\boldsymbol{r}}\left(\tilde{\boldsymbol{q}}_{n}+\varepsilon \delta \tilde{\boldsymbol{q}}\right)=\tilde{\boldsymbol{r}}_{n}+\varepsilon \boldsymbol{L}_{n} \cdot \delta \tilde{\boldsymbol{q}}+O\left(\varepsilon^{2}\right) .
$$

The sequence $\left(\tilde{\boldsymbol{q}}_{0}, \tilde{\boldsymbol{q}}_{1}, \ldots\right)$ converges to the solution of (A 25) if the initial guess is close enough. At each iteration, one computes $\tilde{\boldsymbol{r}}_{n}=\tilde{\boldsymbol{r}}\left(\tilde{\boldsymbol{q}}_{n}\right)$ then solves the linear problem $L_{n} \cdot \delta \tilde{\boldsymbol{q}}_{n}=\tilde{\boldsymbol{r}}_{n}$ for $\delta \tilde{\boldsymbol{q}}_{n}$. We do this by the generalized minimal residual (GMRES) algorithm, which finds an approximation of $\delta \tilde{\boldsymbol{q}}_{n}$ in the subspace of $Q$ spanned by $\tilde{\boldsymbol{r}}_{n}, L_{n} \cdot \tilde{\boldsymbol{r}}_{n}, \ldots, L_{n}^{d} \cdot \tilde{\boldsymbol{r}}_{n}$ so as to minimize the norm of residual $L_{n} \cdot \delta \tilde{\boldsymbol{q}}_{n}-\tilde{\boldsymbol{r}}_{n}$ (Saad \& Schultz 1986). The operator $\boldsymbol{L}_{n}$ enters the algorithm only through operations of the type $\boldsymbol{\beta} \leftarrow \boldsymbol{L}_{n} \cdot \boldsymbol{\alpha}$. As a result the whole calculation combines calculations that all resemble very closely the computation of trends for the temporal problem. In particular, the construction and factorization of a large matrix representing the linearized operator $L_{n}$ is avoided. In principle GMRES should also be more efficient than a direct solution of (A 26), but we make no claim regarding efficiency since we use rather large Krylov spaces $(d=1000)$. We set the relative tolerance of the GMRES solver to $10^{-2}$ and the relative tolerance of the Newton solver to $10^{-5}$. We find it convenient to solve the steady-state equations (2.15) rather than the time-dependent (2.5)-(2.6) because we can obtain the steady state faster and at the same time control the quality of the computed steady state (the residual of the Newton iteration).

Non-stratified travelling rolls are computed first. The computation is bootstrapped at $R e=250$ where the final state of a long temporal simulation provides a good initial guess for the Newton-Krylov iteration. We then progressively increase $R e$, using as an initial guess the travelling roll computed at the immediately smaller $R e$. At fixed $R e$ we progressively increase $R i$. 



FIGURE 13. Numerical convergence at $R e=1000, \operatorname{Pr}=4$ as vertical resolution is increased from $N_{z}=60$ (dotted) to $N_{z}=80$ (solid). (a) Secondary growth rate $\sigma_{2}\left(k_{y}\right)$ (see figure 7) computed with $N_{z}=60$ (dotted) and $N_{z}=80$ (solid). (b) Nonlinear energetics of Ekman rolls (see figure 11), at $R e=1000, \operatorname{Pr}=4, R i=0.015$.

\section{A.6. Numerical parameters and convergence}

In all our calculations we use $N_{x}=64$ and $M_{x}=21$. The vertical resolution is set to $N_{z}=60$. We have checked that no oscillations occur in the computed fields. Furthermore increasing the vertical resolution to $N_{z}=80$ does not change significantly the characteristics of the steady rolls, especially the buoyancy gradient. Specifically, the total energy (figure 4) and maximum negative buoyancy gradient (figure 5) agree within 5-10\% when computed with $N_{z}=80$ or $N_{z}=60$.

Computations of secondary growth rates are not affected by resolution in the $y$ direction since the Fourier modes are not coupled. For these simulations we use $N_{y}=$ 192 and $L_{y}=50 \pi$ for $R i>0.05$, (respectively $L_{y}=40 \pi$ for $R i \leqslant 0.05$ ) which provides a spectral resolution of $\Delta k_{y}=0.04$ and a largest wave vectors $k_{y}^{\max }=64 \Delta k_{y}=2.6$ (respectively $\Delta k_{y}=0.05$ and $k_{y}^{\max }=3.2$ ). The tolerance $\varepsilon$ for the computation of the secondary growth rate is set to $10^{-2}$ except at $\operatorname{Re}=500, \operatorname{Pr}=1, \operatorname{Ri}=0.015$. For this set of parameters the growth rates are very low and the tolerance was raised to 0.04 , otherwise very long simulations would have been required. For the most demanding parameters we consider, i.e. $\operatorname{Re}=1000, \operatorname{Pr}=4$. the maximum growth rate of the secondary instability (occurring around $k_{y}=0.4$ ) varies (as vertical resolution increases from 60 to 80 ) by less than $1 \%$ (figure 13). The secondary maximum of $\sigma_{2}$ 
near $k_{y}=2.2$ varies somewhat more, by $\sim 5 \%$. This is the worst case and convergence is better for $\operatorname{Re}=500$ or $\operatorname{Pr}=4$.

For the nonlinear simulations the domain size along $y$ is equal to the wavelength of the most unstable secondary mode and $N_{y}=32$. We have repeated the nonlinear simulations for $\operatorname{Re}=1000, \operatorname{Pr}=4$ at increased resolution $N_{z}=80$. The main features do not change, especially the shape of the streamlines which we use to discuss the turbulent breakdown of Ekman rolls, but $N_{z}=60$ and $N_{z}=80$ do not yield strictly identical results. This is illustrated in figure 13 which repeats part of figure 11 at both resolutions $N_{z}=60,80$. Although the general picture remains the same, a temporary dip in the kinetic energy around $t=600$ is present at $N_{z}=80$ and absent at $N_{z}=60$. We have taken care to base our analysis only on robust features of the computations.

\section{REFERENCES}

BROWN, R. A. 1970 A secondary flow model for the planetary boundary layer. J. Atmos. Sci 27, 742-757.

BROWN, R. A. 1972 On the inflection point instability of a stratified Ekman boundary layer. J. Atmos. Sci 29, 850-859.

CAldwell, D. R. \& VAN ATtA, C. W. 1970 Characteristics of Ekman boundary layer instabilities. J. Fluid Mech. 44, 79-95.

Caulfield, C. P. \& Peltier, W. R. 2000 The anatomy of the mixing transition in homogeneous and stratified free shear layers. J. Fluid Mech. 413, 1-47.

Chandrasekhar, S. 1961 Hydrodynamic and Hydromagnetic Stability. Clarendon.

CHLOND, A. 1987 A numerical study of horizontal roll vortices in neutral and unstable boundary layers. Beitr. Phys. Atmos. 60, 144-169.

Coleman, G. N. 1999 Similarity statistics from a direct numerical simulation of the neutrally stratified planetary boundary layer. J. Atmos. Sci. 56, 891-900.

Coleman, G. N., Ferziger, J. H. \& Spalart, P. R. 1990 A numerical study of the turbulent Ekman layer. J. Fluid Mech. 213, 313-348.

Coleman, G. N., Ferziger, J. H. \& Spalart, P. R. 1992 Direct simulation of the stably stratified turbulent Ekman layer. J. Fluid Mech. 244, 677-712.

Dubos, T., Barthlott, C. \& Drobinski, P. 2008 Emergence and secondary instability of Ekman layer rolls. J. Atmos. Sci 65, 2326-2342.

Ekman, V. W. 1905 On the influence of the Earth's rotation on ocean currents. Arkiv. Matem. Atr. Fysik 2 (11), 1-54.

ETLing, D. \& BRown, R. A. 1993 Roll vortices in the planetary boundary layer: a review. Boundary-Layer Meteorol. 21, 215-248.

FALler, A. J. \& KAYLOR, R. E. 1966 A numerical study of the instability of the laminar Ekman boundary layer. J. Atmos. Sci. 23, 466-480.

FOSTER, R. C. 1996 An analytic model for planetary boundary layer roll vortices. PhD thesis, University of Washington.

Haeusser, T. M. \& Leibovich, S. 2003 Pattern formation in the marginally unstable Ekman layer. J. Fluid Mech. 479, 125-144.

Howard, L. N. 1961 Note on a paper of John W. Miles. J. Fluid Mech. 10, 509-512.

Julien, S., Ortiz, S. \& ChomaZ, J.-M. 2004 Secondary instability mechanisms in the wake of a flat plate. Eur. J. Mech. (B/Fluids) 23, 157-165.

KAYlor, R. \& FAller, A. J. 1972 Instability of the stratified Ekman boundary layer and the generation of internal waves. J. Atmos. Sci. 29, 497-509.

Klatssen, G. P. \& Peltier, W. R. 1985 The effect of Prandtl number on the evolution and stability of Kelvin-Helmholtz billows. Geophys. Astrophys. Fluid Dyn. 32, 23-60.

Leibovich, S. \& LelE, S. K. 1985 The influence of the horizontal component of Earth's angular velocity on the instability of the Ekman layer. J. Fluid Mech. 150, 41-87.

LiLLY, D. K. 1966 On the instability of Ekman boundary flow. J. Atmos. Sci. 23, 481-494. 
MAHRT, L. 1999 Stratified atmospheric boundary layers. Boundary-Layer Meteorol. 90, 375-396.

Mashayek, A. \& Peltier, W. R. 2012 The 'zoo' of secondary instabilities precursory to stratified shear flow transition. Part 1. Shear aligned convection, pairing, and braid instabilities. J. Fluid Mech. 708, 5-44.

Miles, J. W. 1961 On the stability of heterogeneous shear flows. J. Fluid Mech. 10, 496-508.

Mkhinini, N., Dubos, T \& Drobinski, P. 2013 On the non-linear destabilization of stably stratified shear flow. J. Fluid Mech. (submitted).

Peltier, W. R. \& CAulfield, C. P. 2003 Mixing efficiency in stratified shear flows. Annu. Rev. Fluid Mech. 35, 135-167.

SAAD, Y. \& SCHUlTZ, M. H. 1986 GMRES: a generalized minimal residual algorithm for solving nonsymmetric linear systems. SIAM J. Sci. Comput. 7, 856-869.

SPALART, P. R. 1989 Theoretical and numerical study of a three-dimensional turbulent boundary layer. J. Fluid Mech. 205, 319-340.

Spalart, P. R., Coleman, G. N. \& Johnstone, R. 2008 Direct numerical simulation of the Ekman layer: a step in Reynolds number, and cautious support for a log law with a shifted origin. Phys. Fluids 20 (10), 101507.

Spalart, P. R., Coleman, G. N. \& Johnstone, R. 2009 Retraction: 'direct numerical simulation of the Ekman layer: a step in Reynolds number, and cautious support for a log law with a shifted origin'. Phys. Fluids 21 (10), 109901.

Young, G. S., Kristovich, D. A. R., Huelmfelt, M. R. \& Foster, R. C. 2002 Rolls, streets, waves, and more: a review of quasi-two-dimensional structures in the atmospheric boundary layer. Bull. Am. Meteorol. Soc. 83, 997-1001. 